\title{
REPRESENTATION FORMULA FOR DISCRETE INDEFINITE AFFINE SPHERES
}

\author{
SHIMPEI KOBAYASHI AND NOZOMU MATSUURA
}

\begin{abstract}
We present a representation formula for discrete indefinite affine spheres via loop group factorizations. This formula is derived from the Birkhoff decomposition of loop groups associated with discrete indefinite affine spheres. In particular we show that a discrete indefinite improper affine sphere can be constructed from two discrete plane curves.
\end{abstract}

\section{INTRODUCTION}

Around 1908 Tzitzeica introduced surfaces in [25]-[30], which are now called proper affine spheres with center at the origin, with the property that the Gaussian curvature is proportional to the fourth power of the support function from the origin. He observed that this property is invariant under an affine transformation fixing the origin. This work is regarded as the source of affine differential geometry of surfaces, and gives his name to the structure equation of proper affine spheres. The reader is referred to [23] for an account of the Tzitzeica equation within its classical context of surface theory in equicentroaffine geometry. The Tzitzeica equation is now known to be one of the most famous soliton equations in the theory of integrable systems ([7], [17, [31], [12]). In fact it is obtained by a so-called $B$-type reduction of the 2-dimensional Toda lattice equation ([19]). The proper affine sphere can be understood as an affine geometric analogue of the sphere, in a sense that its affine normals meet at the origin. When the affine normals are parallel, the surface can be regarded as an analogue of the plane, and is called an improper affine sphere. The improper affine sphere is described by the Liouville equation, which is also known to be integrable.

It is a distinctive feature of integrable systems that we can discretize them while keeping their integrability. For example, a discrete Liouville equation was derived in [10] using the bilinear techniques, and the corresponding discrete improper affine sphere was introduced in [16]. As for the Tzitzeica equation, an integrable discrete model was proposed in [3], which can be written into the trilinear equation in terms of the $\tau$ function. Their approaches in finding the discrete equations belong to the theory of discrete differential geometry (DDG), which investigates the geometric objects that are described by integrable partial difference equations, refer to [4] for a comprehensive introduction to DDG. We expect that investigating discrete objects may offer a better understanding way of smooth objects, and as a consequence of it, DDG can be applied to practical use in architecture, computer vision, operations research and so on. See, for instance, [21], [5] and [9].

Date: January 23, 2020.

2010 Mathematics Subject Classification. Primary 53A15, 37K10.

Key words and phrases. affine sphere, Tzitzeica equation, Liouville equation, discrete differential geometry, discrete integrable systems, loop group.

This work was partially supported by JSPS KAKENHI Grant Numbers JP26400059, JP15K04862, JP18K03265 and JP19K03507. The first named author was also supported by Deutsche Forschungsgemeinschaft-Collaborative Research Center, TRR 109, "Discretization in Geometry and Dynamics". The second named author was also supported by the Fukuoka University fund, grant number 177102. 
The interrelations existing between integrable systems and geometry are described by the Gauss-Weingarten formula, because the moving frames of surfaces give the Lax pairs of soliton equations. Besides that, it is the point that we are able to introduce a natural parameter into the Lax pair, which is often called the spectral parameter. Thus we can investigate surfaces from a view point of the loop group theory, which also helps us in deriving discrete counterparts of surfaces. Indeed, on the indefinite affine spheres, the loop group discretization method has been demonstrated in [3]. Further, we can make a use of the Birkhoff decomposition of loop groups so as to give construction methods for special classes of discrete surfaces. For example, discrete counterparts for the surfaces with constant negative curvature can be defined and constructed via loop group method ([20], [13]), where a discrete analogue of separation of variables for sine-Gordon equation ([14]) is presented.

In this paper, we give a construction method for discrete indefinite affine spheres by using a loop group method. In particular we show that a discrete indefinite improper affine sphere can be constructed from two discrete plane curves. The paper is organized as follows: in Section 1, after explaining some basic notions of affine differential geometry, we prepare loop groups associated with affine spheres. We close the section by rephrasing the representation formula by Blaschke for improper affine spheres and illustrating some examples that may have singularities. In Section 2, we discretize the representation formula, so that the discrete improper affine spheres, which may have singularities, are constructed from two planar discrete curves.

\section{INDEFINITE AFFINE SPHERES}

1.1. Preliminaries. Let $f$ be an immersion from a domain $\mathbb{D} \subset \mathbb{R}^{2}$ to the affine space $\left(\mathbb{R}^{3}\right.$, det). Here we use determinant function as a fixed volume element on $\mathbb{R}^{3}$. Let $\xi$ be an transversal vector field to $f$, that is, for each $(x, y) \in \mathbb{D}$ the vector $\xi(x, y)$ never tangent to the surface $f(\mathbb{D})$. A symmetric bilinear function $h=\left[h_{i j}\right]$ is defined by the Gauss formula

$$
\begin{aligned}
\partial_{x}^{2} f & =w_{11}^{1} \partial_{x} f+w_{11}^{2} \partial_{y} f+h_{11} \xi, \\
\partial_{y} \partial_{x} f & =w_{12}^{1} \partial_{x} f+w_{12}^{2} \partial_{y} f+h_{12} \xi, \\
\partial_{y}^{2} f & =w_{22}^{1} \partial_{x} f+w_{22}^{2} \partial_{y} f+h_{22} \xi,
\end{aligned}
$$

where $\partial_{x}=\partial / \partial x$ and $\partial_{y}=\partial / \partial y$. It is easy to check that the rank of $h$ is independent of the choice of $\xi$. If the rank of $h$ is $2, h$ can be treated as a nondegenerate metric on $\mathbb{D}$. This is the basic assumption on which Blaschke [1] developed the affine differential geometry of surfaces. We can define canonical transversal vector field by the properties that the induced volume element on $\mathbb{D}$ coincides with the volume element of the affine metric $h$, namely

$$
\operatorname{det}\left[\partial_{x} f, \partial_{y} f, \xi\right]^{2}=\left|h_{11} h_{22}-\left(h_{12}\right)^{2}\right|,
$$

and both $\partial_{x} \xi$ and $\partial_{y} \xi$ are tangent to $f(\mathbb{D})$. Such a $\xi$ is unique up to sign, and is called the affine normal field. The immersion $f$ with the affine normal field $\xi$ is called the Blaschke immersion, and the map $\tilde{F}: \mathbb{D} \rightarrow \mathrm{GL}_{3} \mathbb{R},(x, y) \mapsto\left[\partial_{x} f, \partial_{y} f, \xi\right]$ is called a moving frame of $f$. It is known that, for a Blaschke immersion $f$, half of the Laplacian (1/2) $\Delta f$ relative to the affine metric $h$ is equal to the affine normal field $\xi$.

For a Blaschke immersion $f$, the affine shape operator $s=\left[s_{i j}\right]$ is defined by the Weingarten formula

$$
\begin{aligned}
& \partial_{x} \xi=-s_{11} \partial_{x} f-s_{21} \partial_{y} f, \\
& \partial_{y} \xi=-s_{12} \partial_{x} f-s_{22} \partial_{y} f .
\end{aligned}
$$


If the affine shape operator $s$ is proportional to the identity, that is $s=H$ id, then the Blaschke immersion $f$ is called an affine sphere. By virtue of the integrability condition, this function $H$ should be a constant. If $H=0$ then $f$ is called an improper affine sphere, and if $H \neq 0$ then $f$ is called a proper affine sphere. On use of a scaling transformation of the ambient space and a change of the orientation $f \mapsto-f$, we can normalize the constant $H$ to be -1 if $H \neq 0$. For example, a graph immersion

$$
f(x, y)=\left[\begin{array}{c}
x \\
y \\
\psi(x, y)
\end{array}\right]
$$

is an improper affine sphere with the constant affine normal field $\xi(x, y)={ }^{\mathrm{t}}[0,0,1]$ if and only if $\psi$ satisfies the Monge-Ampère equation

$$
\left(\partial_{x}^{2} \psi\right)\left(\partial_{y}^{2} \psi\right)-\left(\partial_{x} \partial_{y} \psi\right)^{2}= \pm 1
$$

In general, if $f$ is an improper affine sphere, then the affine normals are parallel in $\mathbb{R}^{3}$. If $f$ is a proper affine sphere, then the affine normals meet at one point in $\mathbb{R}^{3}$, which is called the center.

Let $f$ be an affine sphere whose affine metric $h$ has signature $(+,-)$. We call such an $f$ indefinite affine sphere in short. The affine normal field may be expressed as

$$
\xi=-H f+(1+H) \xi_{0},
$$

where $H \in\{-1,0\}$ and $\xi_{0}$ is a constant vector. By an appropriate affine transformation on $\mathbb{R}^{3}$ we can fix $\xi_{0}$ to be ${ }^{\mathrm{t}}[0,0,1]$. We shall employ the asymptotic coordinate systems with respect to $h$, and substitute the symbols $(x, y)$ with $(u, v)$. As far as $\operatorname{det} \tilde{F} \neq 0$, without loss of generality we can assume that $\operatorname{det} \tilde{F}>0$, and define three functions $\omega, A, B$ by

$$
\omega=\operatorname{det} \tilde{F}, \quad A=\operatorname{det}\left[\partial_{u} f, \partial_{u}^{2} f, \xi\right], \quad B=\operatorname{det}\left[\partial_{v}^{2} f, \partial_{v} f, \xi\right] .
$$

We rewrite the Gauss-Weingarten formulas as

$$
\partial_{u} \tilde{F}=\tilde{F}\left[\begin{array}{ccc}
\partial_{u} \log \omega & 0 & -H \\
A \omega^{-1} & 0 & 0 \\
0 & \omega & 0
\end{array}\right], \quad \partial_{v} \tilde{F}=\tilde{F}\left[\begin{array}{ccc}
0 & B \omega^{-1} & 0 \\
0 & \partial_{v} \log \omega & -H \\
\omega & 0 & 0
\end{array}\right] .
$$

The compatibility condition between these two equations, namely $\partial_{u} \partial_{v} \tilde{F}=\partial_{v} \partial_{u} \tilde{F}$, is given by the three partial differential equations

$$
\begin{gathered}
\partial_{v} \partial_{u} \log \omega+A B \omega^{-2}+H \omega=0, \\
\partial_{v} A=0, \quad \partial_{u} B=0 .
\end{gathered}
$$

The equations in (1.5) are clearly solved as $A=A(u)$ and $B=B(v)$ respectively. Equation (1.4) is called the Tzitzeica equation if $H=-1$, and the Liouville equation if $H=0$. It is known that general solutions to the Liouville equation are given by two real functions of one variable, see the formula $(1.29)$ in Remark 1.7 .

Conversely, a triad $(\omega, A, B)$ of solutions to the system $(1.4)-(1.5)$, or in other words, a pair of the affine metric $h=2 \omega d u d v$ and the cubic form $C=A d u^{3}+B d v^{3}$, gives a unique indefinite affine sphere up to equiaffine transformations. Since the system (1.4) - 1.5) is invariant under a transformation

$$
A \mapsto \lambda^{3} A, \quad B \mapsto \lambda^{-3} B, \quad \lambda \in \mathbb{R}^{\times}=\mathbb{R} \backslash\{0\},
$$

if $(\omega, A, B)$ is a triad of solutions to $1.4-1.5)$ then $\left(\omega, \lambda^{3} A, \lambda^{-3} B\right)$ is also a triad of solutions to the same system. Therefore, there exists a family of indefinite affine spheres $\left\{f^{\lambda}\right\}$ that is parametrized by $\lambda \in \mathbb{R}^{\times}$, and $f^{1}$ is the original affine sphere $f$. This 1 -parameter family of 
indefinite affine spheres, which we call the associated family of $f$, has the property that they have the same affine metric and the same constant affine mean curvature $(1 / 2) \operatorname{tr} s=H$.

\subsection{Loop group description. We define a gauged frame $F$ of $f^{\lambda}$ by}

$$
F=\left[\partial_{u} f^{\lambda}, \partial_{v} f^{\lambda}, \xi^{\lambda}\right] \operatorname{diag}\left(\lambda^{-1} \omega^{-1 / 2}, \lambda \omega^{-1 / 2}, 1\right),
$$

where $\xi^{\lambda}=-H f^{\lambda}+(1+H) \xi_{0}$. For any $((u, v), \lambda) \in \mathbb{D} \times \mathbb{R}^{\times}$, the frame $F$ takes values in the special linear group $\mathrm{SL}_{3} \mathbb{R}$ and satisfies the partial differential equations

$$
\partial_{u} F=F U, \quad \partial_{v} F=F V
$$

where

$$
\begin{aligned}
U & =\left[\begin{array}{ccc}
(1 / 2) \partial_{u} \log \omega & 0 & -\lambda H \omega^{1 / 2} \\
\lambda A \omega^{-1} & -(1 / 2) \partial_{u} \log \omega & 0 \\
0 & \lambda \omega^{1 / 2} & 0
\end{array}\right], \\
V & =\left[\begin{array}{ccc}
-(1 / 2) \partial_{v} \log \omega & \lambda^{-1} B \omega^{-1} & 0 \\
0 & (1 / 2) \partial_{v} \log \omega & -\lambda^{-1} H \omega^{1 / 2} \\
\lambda^{-1} \omega^{1 / 2} & 0 & 0
\end{array}\right] .
\end{aligned}
$$

By multiplying $F$ by some constant matrix from the left if necessary, we can assume that

$$
F(0,0, \lambda)=\text { id }
$$

at the base point $(u, v)=(0,0)$. The gauged frame $F$ which satisfies the system $(1.7)-(1.8)$ with initial condition $(1.9)$ will be called the extended frame of an indefinite affine sphere $f$.

Moreover one can check that the matrices $U=U(\lambda)$ and $V=V(\lambda)$ in $(1.8)$ satisfy

$$
\begin{aligned}
-{ }^{\mathrm{t}} U(-\lambda) T & =T U(\lambda), \quad-{ }^{\mathrm{t}} V(-\lambda) T=T V(\lambda), \\
U(q \lambda) & =Q U(\lambda) Q^{-1}, \quad V(q \lambda)=Q V(\lambda) Q^{-1},
\end{aligned}
$$

where

$$
T=\left[\begin{array}{ccc}
0 & 1 & 0 \\
1 & 0 & 0 \\
0 & 0 & -H
\end{array}\right], \quad Q=\operatorname{diag}\left(q, q^{2}, 1\right), \quad q=e^{2 \pi \sqrt{-1} / 3}
$$

Therefore $F$ must satisfy

$$
\begin{aligned}
{ }^{\mathrm{t}} F(-\lambda){ }^{-1} T & =T F(\lambda), \\
F(q \lambda) & =Q F(\lambda) Q^{-1},
\end{aligned}
$$

and hence the loop algebra and the loop group can be introduced ([8]) as

$$
\begin{aligned}
\Lambda \mathfrak{s l}_{3} \mathbb{R} & =\left\{\Phi: \mathbb{S}^{1} \rightarrow \mathfrak{s l}_{3} \mathbb{C} \mid \overline{\Phi(\bar{\lambda})}=\Phi(\lambda),{ }^{-}{ }^{\mathrm{t}} \Phi(-\lambda) T=T \Phi(\lambda), \Phi(q \lambda)=Q \Phi(\lambda) Q^{-1}\right\}, \\
\Lambda \mathrm{SL}_{3} \mathbb{R} & =\left\{\phi: \mathbb{S}^{1} \rightarrow \mathrm{SL}_{3} \mathbb{C} \mid \overline{\phi(\bar{\lambda})}=\phi(\lambda),{ }^{\mathrm{t}} \phi(-\lambda)^{-1} T=T \phi(\lambda), \phi(q \lambda)=Q \phi(\lambda) Q^{-1}\right\} .
\end{aligned}
$$

Here the overlines mean complex conjugate, and $\mathfrak{s l}_{3} \mathbb{C}$ is the Lie algebra of $\mathrm{SL}_{3} \mathbb{C}$, that is, $\mathfrak{s l}_{3} \mathbb{C}$ is the set of trace-free matrices. It should be noted that the extended frame $F$ is $\Lambda \mathrm{SL}_{3} \mathbb{R}$ valued function on $\mathbb{D}$, because $F$, which is originally defined on $\lambda \in \mathbb{R}^{\times}$, can be analytically extended to $\mathbb{C}^{\times}$. The subgroups

$$
\begin{aligned}
& \Lambda^{+} \mathrm{SL}_{3} \mathbb{R}=\left\{\phi \in \Lambda \mathrm{SL}_{3} \mathbb{R} \mid \phi(\lambda)=\sum_{k=0}^{\infty} \lambda^{k} \phi_{k}\right\}, \\
& \Lambda^{-} \mathrm{SL}_{3} \mathbb{R}=\left\{\phi \in \Lambda \mathrm{SL}_{3} \mathbb{R} \mid \phi(\lambda)=\sum_{k=0}^{\infty} \lambda^{-k} \phi_{k}\right\}
\end{aligned}
$$


will play important roles in the following discussions, together with

$$
\begin{aligned}
& \Lambda_{*}^{+} \mathrm{SL}_{3} \mathbb{R}=\left\{\phi \in \Lambda^{+} \mathrm{SL}_{3} \mathbb{R} \mid \phi(\lambda)=\mathrm{id}+\sum_{k=1}^{\infty} \lambda^{k} \phi_{k}\right\}, \\
& \Lambda_{*}^{-} \mathrm{SL}_{3} \mathbb{R}=\left\{\phi \in \Lambda^{-} \mathrm{SL}_{3} \mathbb{R} \mid \phi(\lambda)=\mathrm{id}+\sum_{k=1}^{\infty} \lambda^{-k} \phi_{k}\right\} .
\end{aligned}
$$

Similarly subalgebras $\Lambda^{+} \mathfrak{s l}_{3} \mathbb{R}, \Lambda^{-} \mathfrak{s l}_{3} \mathbb{R}$ and $\Lambda_{*}^{+} \mathfrak{s l}_{3} \mathbb{R}, \Lambda_{*}^{-} \mathfrak{s l}_{3} \mathbb{R}$ are defined. We note that $\Phi_{ \pm} \in$ $\Lambda_{*}^{ \pm} \mathfrak{s l}_{3} \mathbb{R}$ have the expansion $\Phi_{ \pm}(\lambda)=\sum_{k=1}^{\infty} \lambda^{ \pm k} \Phi_{k}$, where double signs correspond.

Proposition 1.1. Let $F(\lambda)=\sum_{k=-\infty}^{\infty} \lambda^{k} F_{k} \in \Lambda \mathrm{SL}_{3} \mathbb{C}$ satisfy the twisted condition (1.13). Then coefficient matrices of $F(\lambda)$ are of the form

$$
F_{3 l}=\operatorname{diag}(*, *, *), \quad F_{3 l+1}=\left[\begin{array}{ccc}
0 & 0 & * \\
* & 0 & 0 \\
0 & * & 0
\end{array}\right], \quad F_{3 l+2}=\left[\begin{array}{ccc}
0 & * & 0 \\
0 & 0 & * \\
* & 0 & 0
\end{array}\right]
$$

for all integers $l$.

Proof. Because $Q F(\lambda) Q^{-1}=\sum_{k \in \mathbb{Z}} \lambda^{k} Q F_{k} Q^{-1}$ and

$$
F(q \lambda)=\sum_{k \in \mathbb{Z}} \lambda^{k} q^{k} F_{k}=\sum_{l \in \mathbb{Z}}\left(\lambda^{3 l} F_{3 l}+\lambda^{3 l+1} q F_{3 l+1}+\lambda^{3 l+2} q^{2} F_{3 l+2}\right),
$$

we have $F_{3 l}=Q F_{3 l} Q^{-1}, q F_{3 l+1}=Q F_{3 l+1} Q^{-1}$, and $q^{2} F_{3 l+2}=Q F_{3 l+2} Q^{-1}$.

We now recall Birkhoff decomposition theorem for the loop group $\Lambda \mathrm{SL}_{3} \mathbb{R}$.

Theorem 1.2 (Birkhoff decomposition [8], 22]). The respective multiplication maps

$$
\Lambda_{*}^{+} \mathrm{SL}_{3} \mathbb{R} \times \Lambda^{-} \mathrm{SL}_{3} \mathbb{R} \rightarrow \Lambda \mathrm{SL}_{3} \mathbb{R} \quad \text { and } \quad \Lambda_{*}^{-} \mathrm{SL}_{3} \mathbb{R} \times \Lambda^{+} \mathrm{SL}_{3} \mathbb{R} \rightarrow \Lambda \mathrm{SL}_{3} \mathbb{R}
$$

are diffeomorphisms onto its images. Moreover, the images $\Lambda_{*}^{+} \mathrm{SL}_{3} \mathbb{R} \cdot \Lambda^{-} \mathrm{SL}_{3} \mathbb{R}$ and $\Lambda_{*}^{-} \mathrm{SL}_{3} \mathbb{R}$. $\Lambda^{+} \mathrm{SL}_{3} \mathbb{R}$ are both open and dense in $\Lambda \mathrm{SL}_{3} \mathbb{R}$, which will be called the big cells.

Roughly speaking, Theorem 1.2 says that for almost all $g \in \Lambda \mathrm{SL}_{3} \mathbb{R}$, there uniquely exist pairs $\left(g_{+}, g_{-}\right) \in \Lambda_{*}^{+} \mathrm{SL} 3 \mathbb{R} \times \Lambda^{-} \mathrm{SL}_{3} \mathbb{R}$ and $\left(\tilde{g}_{-}, \tilde{g}_{+}\right) \in \Lambda_{*}^{-} \mathrm{SL}_{3} \mathbb{R} \times \Lambda^{+} \mathrm{SL}_{3} \mathbb{R}$ such that

$$
g=g_{+} g_{-}=\tilde{g}_{-} \tilde{g}_{+} \cdot
$$

The following theorem has been proven for indefinite proper affine spheres $(H=-1)$ in $[8$, Proposition 5.2 and Theorems 7.1, 6.1]. Here we show a proof which is valid for both $H=-1$ or $H=0$.

Theorem 1.3. Let $f$ be an indefinite affine sphere, and $(u, v) \in \mathbb{D}$ be its asymptotic coordinates. Consider the Birkhoff decompositions for the extended frame $F$ near $(u, v)=(0,0)$ as

$$
F=F_{+} F_{-}=G_{-} G_{+},
$$

where $F_{+} \in \Lambda_{*}^{+} \mathrm{SL}_{3} \mathbb{R}, F_{-} \in \Lambda^{-} \mathrm{SL}_{3} \mathbb{R}, G_{+} \in \Lambda^{+} \mathrm{SL}_{3} \mathbb{R}$ and $G_{-} \in \Lambda_{*}^{-} \mathrm{SL}_{3} \mathbb{R}$. Then $F_{+}$and $G_{-}$ do not depend on $v$ and $u$ respectively, and their Maurer-Cartan forms are given as

$$
F_{+}^{-1} d F_{+}=\xi_{+}, \quad G_{-}^{-1} d G_{-}=\xi_{-},
$$

where

$$
\xi_{+}=\lambda\left[\begin{array}{ccc}
0 & 0 & -H \alpha \\
\beta & 0 & 0 \\
0 & \alpha & 0
\end{array}\right] d u, \quad \xi_{-}=\lambda^{-1}\left[\begin{array}{ccc}
0 & \sigma & 0 \\
0 & 0 & -H \rho \\
\rho & 0 & 0
\end{array}\right] d v .
$$

Here the functions $\alpha, \beta$ depend only on $u$, and $\rho, \sigma$ only on $v$. Moreover, $\alpha$ and $\rho$ have no zeros near the base point $(u, v)=(0,0)$. 
Conversely, let $\left(\xi_{+}, \xi_{-}\right)$be a pair of 1 -forms as (1.17), and $\left(F_{+}, G_{-}\right)$be a pair of solutions to the linear ordinary differential equations

$$
d F_{+}=F_{+} \xi_{+}, \quad d G_{-}=G_{-} \xi_{-}
$$

with the initial condition $F_{+}(0, \lambda)=G_{-}(0, \lambda)=\mathrm{id}$. Define $V_{+} \in \Lambda_{*}^{+} \mathrm{SL}_{3} \mathbb{R}$ and $V_{-} \in \Lambda^{-} \mathrm{SL}_{3} \mathbb{R}$ by the Birkhoff decomposition for $G_{-}^{-1} F_{+}$near $(u, v)=(0,0)$ as

$$
G_{-}^{-1} F_{+}=V_{+} V_{-}^{-1},
$$

and write $\hat{F}=F_{+} V_{-}=G_{-} V_{+}$. Then there exists a diagonal matrix $D=\operatorname{diag}\left(d, d^{-1}, 1\right)$ with some non-vanishing function $d=d(u, v)$ such that $D_{0}{ }^{-1} \hat{F} D$, where $D_{0}=\left.D\right|_{(u, v)=(0,0)}$, is the extended frame of an indefinite affine sphere $f$ with the cubic differential $C=\alpha^{2} \beta d u^{3}+$ $\rho^{2} \sigma d v^{3}$. In particular, in case of proper affine spheres $(H=-1)$, the third column of $D_{0}{ }^{-1} \hat{F} D$ directly gives the position vector of $f$.

Remark 1.4. The pair of 1-forms defined in (1.17) will be called the pair of normalized potentials for an indefinite affine sphere. It should be noted that the resulting indefinite affine sphere which is constructed from a pair of normalized potentials would have singularities where $G_{-}^{-1} F_{+}$is outside of the big-cell for the Birkhoff decomposition.

Proof. Let $F$ be an extended frame, and define $F_{+}$and $F_{-}$by 1.15 . Therefore we have $F_{+}=F F_{-}^{-1}$ and so that

$$
F_{+}^{-1} \partial_{v} F_{+}=F_{-} F^{-1} \partial_{v}\left(F F_{-}^{-1}\right)=\left(F_{-} V-\partial_{v} F_{-}\right) F_{-}^{-1},
$$

where $V$ is given by (1.8). Since $V$ takes values in $\Lambda^{-} \mathfrak{s l}_{3} \mathbb{R}$, the right-hand side takes values in it. Moreover since $F_{+}$takes values in $\Lambda_{*}^{+} \mathrm{SL}_{3} \mathbb{R}$, the left-hand side takes values in $\Lambda_{*}^{+} \mathfrak{s l}_{3} \mathbb{R}$. Thus we have $F_{+}^{-1} \partial_{v} F_{+}=0$, which shows that $F_{+}$does not depend on $v$. Similarly $\partial_{u} G_{-}=0$.

Next, we compute $F_{+}^{-1} d F_{+}$and $G_{-}^{-1} d G_{-}$. We have

$$
F_{+}^{-1} d F_{+}=F_{-} F^{-1} d\left(F F_{-}^{-1}\right)=\left(F_{-} U-\partial_{u} F_{-}\right) F_{-}^{-1} d u
$$

where $U$ is given by (1.8). Since $U$ has the form $U=U^{0}+\lambda U^{1}$ and $F_{-}$takes values in $\Lambda^{-} \mathrm{SL}_{3} \mathbb{R}$, we have

$$
\xi_{+}=F_{+}^{-1} d F_{+}=\left(X^{0}+\lambda X^{1}\right) d u .
$$

Here $X^{0}=0$ because $\xi_{+}$should be a $\Lambda_{*}^{+} \mathfrak{s l}_{3} \mathbb{R}$-valued 1-form. The twisted condition (1.11) implies that $X^{1}$ have the form

$$
X^{1}=\left[\begin{array}{ccc}
0 & 0 & x_{13} \\
x_{21} & 0 & 0 \\
0 & x_{32} & 0
\end{array}\right],
$$

where $x_{i j}$ are some functions in $u$. Further the twisted condition 1.10 implies that $x_{13}=$ $-H x_{32}$. Thus we have (1.17) on setting $\alpha=x_{32}$ and $\beta=x_{21}$. If we write $F_{-}$and its inverse as

$$
F_{-}=I^{0}+\lambda^{-1} I^{1}+\cdots, \quad F_{-}^{-1}=J^{0}+\lambda^{-1} J^{1}+\cdots,
$$

where id $=F_{-} F_{-}^{-1}=I^{0} J^{0}+\lambda^{-1}\left(I^{0} J^{1}+I^{1} J^{0}\right)+\cdots$, then we in particular have $I^{0} J^{0}=$ id. Further, from the twisted conditions (1.13) and (1.12), it follows that

$$
I^{0}=\operatorname{diag}\left(i, i^{-1}, 1\right), \quad J_{6}^{0}=\operatorname{diag}\left(i^{-1}, i, 1\right),
$$


where $i$ is some function in $(u, v)$ with no zeros. Noticing that $\lambda X^{1}=\left(F_{-} U-\partial_{u} F_{-}\right) F_{-}^{-1}$, it is easy to see that $X^{1}$ is computed as

$$
X^{1}=I^{0} U^{1} J^{0}=\left[\begin{array}{ccc}
0 & 0 & -H i \omega^{1 / 2} \\
A i^{-2} \omega^{-1} & 0 & 0 \\
0 & i \omega^{1 / 2} & 0
\end{array}\right],
$$

which shows that $\alpha=x_{32}=i \omega^{1 / 2}$ has no zeros. Similarly we can show that $\rho$ has no zeros.

Conversely let $F_{+}$and $G_{-}$be the solutions of $(1.18)$ with initial condition $F_{+}(0, \lambda)=$ $G_{-}(0, \lambda)=$ id and consider the Birkhoff decomposition near $(u, v)=(0,0)$ as $(1.19)$ with $V_{+} \in \Lambda_{*}^{+} \mathrm{SL}_{3} \mathbb{R}$ and $V_{-} \in \Lambda^{-} \mathrm{SL}_{3} \mathbb{R}$. Then the Maurer-Cartan form of $\hat{F}=F_{+} V_{-}=G_{-} V_{+}$is computed as

$$
\begin{aligned}
& \hat{F}^{-1} d \hat{F}=\left(F_{+} V_{-}\right)^{-1} d\left(F_{+} V_{-}\right)=V_{-}^{-1}\left(\xi_{+} V_{-}+d V_{-}\right), \\
& \hat{F}^{-1} d \hat{F}=\left(G_{-} V_{+}\right)^{-1} d\left(G_{-} V_{+}\right)=V_{+}^{-1}\left(\xi_{-} V_{+}+d V_{+}\right) .
\end{aligned}
$$

We write

$$
V_{-}=K_{0}+\lambda^{-1} K_{1}+\cdots, \quad V_{-}^{-1}=L_{0}+\lambda^{-1} L_{1}+\cdots
$$

with the matrices

$$
K_{0}=\operatorname{diag}\left(k, k^{-1}, 1\right), \quad L_{0}=\operatorname{diag}\left(k^{-1}, k, 1\right),
$$

where $k$ is some function in $(u, v)$ which has no zeros. Noticing that $V_{+}$is $\Lambda_{*}^{+} \mathrm{SL}_{3} \mathbb{R}$-valued, it follows from $1.20-1.21$ that $\hat{F}^{-1} d \hat{F}$ is given by

$$
\begin{aligned}
& \hat{F}^{-1} \partial_{u} \hat{F}=\lambda L_{0}\left[\begin{array}{ccc}
0 & 0 & -H \alpha \\
\beta & 0 & 0 \\
0 & \alpha & 0
\end{array}\right] K_{0}, \\
& \hat{F}^{-1} \partial_{v} \hat{F}=\lambda^{-1}\left[\begin{array}{ccc}
0 & \sigma & 0 \\
0 & 0 & -H \rho \\
\rho & 0 & 0
\end{array}\right]+L_{0} \partial_{v} K_{0} .
\end{aligned}
$$

We introduce a gauge $D=\operatorname{diag}\left(d, d^{-1}, 1\right)$, then $F=\hat{F} D$ satisfies that

$$
\begin{aligned}
& F^{-1} \partial_{u} F=\lambda\left[\begin{array}{ccc}
0 & 0 & -H \alpha k^{-1} d^{-1} \\
\beta k^{2} d^{2} & 0 & 0 \\
0 & \alpha k^{-1} d^{-1} & 0
\end{array}\right]+\left[\begin{array}{ccc}
d^{-1} \partial_{u} d & 0 & 0 \\
0 & -d^{-1} \partial_{u} d & 0 \\
0 & 0 & 0
\end{array}\right], \\
& F^{-1} \partial_{v} F=\lambda^{-1}\left[\begin{array}{ccc}
0 & \sigma d^{-2} & 0 \\
0 & 0 & -H \rho d \\
\rho d & 0 & 0
\end{array}\right]+\left[\begin{array}{ccc}
d^{-1} \partial_{v} d+k^{-1} \partial_{v} k & 0 & 0 \\
0 & -d^{-1} \partial_{v} d-k^{-1} \partial_{v} k & 0 \\
0 & 0 & 0
\end{array}\right] .
\end{aligned}
$$

We define $\omega, A, B$ by

$$
\omega=\frac{\alpha \rho}{k}, \quad A=\alpha^{2} \beta, \quad B=\rho^{2} \sigma .
$$

If necessary changing $u \rightarrow-u$ and/or $v \rightarrow-v$, we can assume $\omega>0$, and choose $d=\rho^{-1} \omega^{1 / 2}$. It is easy to check that these matrices $F^{-1} \partial_{u} F$ and $F^{-1} \partial_{v} F$ coincide with (1.8). Thus $D_{0}{ }^{-1} F$, where $D_{0}=\left.D\right|_{(u, v)=(0,0)}$, satisfies $D_{0}^{-1} F(0,0, \lambda)=\mathrm{id}$, and hence is the extended frame of some indefinite affine sphere. 
1.3. Indefinite improper affine spheres. When $H=0$, there exists an integral formula in terms of four functions of one variable, which is known as the Blaschke representation. We first show a fundamental lemma.

Lemma 1.5. Let $H=0$. Then the pair of solutions $\left(F_{+}, G_{-}\right)$to the system (1.16) and (1.17) with the initial condition $F_{+}(0, \lambda)=G_{-}(0, \lambda)=$ id is explicitly given by

$$
F_{+}=\left[\begin{array}{ccc}
1 & 0 & 0 \\
\lambda b & 1 & 0 \\
\lambda^{2} c & \lambda a & 1
\end{array}\right], \quad G_{-}=\left[\begin{array}{ccc}
1 & \lambda^{-1} s & 0 \\
0 & 1 & 0 \\
\lambda^{-1} r & \lambda^{-2} t & 1
\end{array}\right]
$$

where $a, b, c, r, s, t$ are defined as

$$
\begin{aligned}
& a(u)=\int_{0}^{u} \alpha(k) d k, \quad b(u)=\int_{0}^{u} \beta(k) d k, \quad c(u)=\int_{0}^{u} a(k) \beta(k) d k, \\
& r(v)=\int_{0}^{v} \rho(k) d k, \quad s(v)=\int_{0}^{v} \sigma(k) d k, \quad t(v)=\int_{0}^{v} r(k) \sigma(k) d k .
\end{aligned}
$$

Moreover, if $1-b s \neq 0$, then $V_{+} \in \Lambda_{*}^{+} \mathrm{SL}_{3} \mathbb{R}$ and $V_{-} \in \Lambda^{-} \mathrm{SL}_{3} \mathbb{R}$, defined by the Birkhoff decomposition of $G_{-}^{-1} F_{+}$as 1.19 , are given as

$$
\begin{aligned}
V_{+}= & {\left[\begin{array}{ccc}
1 & 0 & 0 \\
\lambda b(1-b s)^{-1} & 1 & 0 \\
\lambda^{2} c(1-b s)^{-1} & \lambda(a(1-b s)+c s) & 1
\end{array}\right], } \\
V_{-}= & {\left[\begin{array}{ccc}
(1-b s)^{-1} & \lambda^{-1} s & 0 \\
0 & 1-b s & 0 \\
\lambda^{-1}\left(r+b t(1-b s)^{-1}\right) & \lambda^{-2} t & 1
\end{array}\right] . }
\end{aligned}
$$

Proof. It is easy to check that $F_{+}, G_{-}$in $(1.22)$ satisfy $(1.16)$ and $(1.17)$ with $H=0$, and $F_{+}(0, \lambda)=G_{-}(0, \lambda)=$ id. The loops $V_{+}, V_{-}$in (1.25), (1.26) clearly belong to $\Lambda_{*}^{+} \mathrm{SL}_{3} \mathbb{R}$, $\Lambda^{-} \mathrm{SL}_{3} \mathbb{R}$ respectively. Because $F_{+} V_{-}=G_{-} V_{+}$, the decomposition 1.19 holds.

The condition $1-b s \neq 0$ in Lemma 1.5 means that $G_{-}^{-1} F_{+}$belongs to the big cell of the Birkhoff decomposition.

Theorem 1.6. Let $\alpha, \beta, \rho, \sigma$ be functions in one variable, and define $a, b, c, r, s, t$ by (1.23) and (1.24). Let $F_{+}, G_{-}, V_{+}, V_{-}$be the loops given by (1.22), (1.25), (1.26), and define $\hat{F}$ by $\hat{F}=F_{+} V_{-}=G_{-} V_{+}$. We assume that $\alpha, \rho, 1-b$ s have no zeros. Then there exists a diagonal matrix $D=\operatorname{diag}\left(d, d^{-1}, 1\right)$ with some function $d$ such that $D_{0}{ }^{-1} \hat{F} D$, where $D_{0}=\left.D\right|_{(u, v)=(0,0)}$, is the extended frame of some indefinite improper affine sphere $f$. The data solving the integrability condition (1.4) -1.5 with $H=0$ are given as

$$
\omega=(1-b s) \alpha \rho, \quad A=\alpha^{2} \beta, \quad B=\rho^{2} \sigma .
$$

Moreover, the associated family of $f$ is given by the representation formula

$$
f^{\lambda}=\left[\begin{array}{c}
\lambda a+\lambda^{-2}(r s-t) \\
\lambda^{2}(a b-c)+\lambda^{-1} r \\
a r-(a b-c)(r s-t)+\lambda^{3} \int_{0}^{u} \alpha(k) c(k) d k+\lambda^{-3} \int_{0}^{v} \rho(k) t(k) d k
\end{array}\right]
$$

where $\lambda \in \mathbb{R}^{\times}$. All indefinite improper affine spheres are locally constructed in this way. 
Proof. First a straightforward computation shows that Maurer-Cartan form of $\hat{F}$ is given by

$$
\begin{aligned}
& \hat{F}^{-1} \partial_{u} \hat{F}=V_{+}^{-1} \partial_{u} V_{+}=\left[\begin{array}{ccc}
0 & 0 & 0 \\
\lambda \beta(1-b s)^{-2} & 0 & 0 \\
0 & \lambda \alpha(1-b s) & 0
\end{array}\right]=\hat{U}, \\
& \hat{F}^{-1} \partial_{v} \hat{F}=V_{-}^{-1} \partial_{v} V_{-}=\left[\begin{array}{ccc}
b \sigma(1-b s)^{-1} & \lambda^{-1} \sigma & 0 \\
0 & -b \sigma(1-b s)^{-1} & 0 \\
\lambda^{-1} \rho & 0 & 0
\end{array}\right]=\hat{V} .
\end{aligned}
$$

Next we take a diagonal gauge $D=\operatorname{diag}\left(d, d^{-1}, 1\right)$. Then the Maurer-Cartan form of $F=\hat{F} D$ is given by

$$
\begin{aligned}
& F^{-1} \partial_{u} F=D^{-1}\left(\hat{U} D+\partial_{u} D\right)=\left[\begin{array}{ccc}
\partial_{u} \log d & 0 & 0 \\
\lambda \beta d^{2}(1-b s)^{-2} & -\partial_{u} \log d & 0 \\
0 & \lambda \alpha d^{-1}(1-b s) & 0
\end{array}\right], \\
& F^{-1} \partial_{v} F=D^{-1}\left(\hat{V} D+\partial_{v} D\right)=\left[\begin{array}{ccc}
b \sigma(1-b s)^{-1}+\partial_{v} \log d & \lambda^{-1} \sigma d^{-2} & 0 \\
0 & -b \sigma(1-b s)^{-1}-\partial_{v} \log d & 0 \\
\lambda^{-1} \rho d & 0 & 0
\end{array}\right] .
\end{aligned}
$$

If necessary, changing $u \rightarrow-u$ and/or $v \rightarrow-v$, we can assume $(1-b s) \alpha \rho>0$. Then setting 1.27 and

$$
d=\frac{\sqrt{(1-b s) \alpha \rho}}{\rho}=\frac{\omega^{1 / 2}}{\rho},
$$

this system accords with (1.7) -1.8 . To obtain the representation formula (1.28), we consider an another diagonal gauge $D=\operatorname{diag}\left(\lambda \omega^{1 / 2}, \lambda^{-1} \omega^{1 / 2}, 1\right)$ as introduced in (1.6). Therefore $\tilde{F}=F \tilde{D}$ satisfies that

$$
\tilde{F}^{-1} \partial_{u} \tilde{F}=\left[\begin{array}{ccc}
\partial_{u} \log \omega & 0 & 0 \\
\lambda^{3} A \omega^{-1} & 0 & 0 \\
0 & \omega & 0
\end{array}\right], \quad \tilde{F}^{-1} \partial_{v} \tilde{F}=\left[\begin{array}{ccc}
0 & \lambda^{-3} B \omega^{-1} & 0 \\
0 & \partial_{v} \log \omega & 0 \\
\omega & 0 & 0
\end{array}\right] .
$$

Thus $\tilde{F}$ is a family of moving frames of indefinite improper affine spheres. The moving frame $\tilde{F}$ can be computed explicitly as

$$
\tilde{F}=G_{-} V_{+} D \tilde{D}=\left[\begin{array}{ccc}
\lambda \alpha & \lambda^{-2} \rho s & 0 \\
\lambda^{2} \alpha b & \lambda^{-1} \rho & 0 \\
\left(\lambda^{3} c+b t+r(1-b s)\right) \alpha & \left(\lambda^{-3} t+c s+a(1-b s)\right) \rho & 1
\end{array}\right] .
$$

Since the moving frame is defined by $\tilde{F}=\left[\partial_{u} f^{\lambda}, \partial_{v} f^{\lambda}, \xi_{0}\right]$, we integrate the first column of $\tilde{F}$ by $u$ and have

$$
f^{\lambda}=\left[\begin{array}{c}
\lambda a \\
\lambda^{2}(a b-c) \\
a r-(r s-t)(a b-c)+\lambda^{3} \int_{0}^{u} \alpha(k) c(k) d k
\end{array}\right]+\left[\begin{array}{l}
x \\
y \\
z
\end{array}\right],
$$

where $x, y, z$ are some functions in $v$. Therefore from the second column of $\tilde{F}$, we have

$$
\left[\begin{array}{c}
\lambda^{-2} \rho s \\
\lambda^{-1} \rho \\
\left(\lambda^{-3} t+c s+a(1-b s)\right) \rho
\end{array}\right]=\partial_{v} f^{\lambda}=\left[\begin{array}{c}
x^{\prime} \\
y^{\prime} \\
a r^{\prime}-(r s-t)^{\prime}(a b-c)+z^{\prime}
\end{array}\right] .
$$

Therefore

$$
x=\lambda^{-2}(r s-t), \quad y=\lambda^{-1} r, \quad z=\lambda^{-3} \int_{0}^{v} \rho(k) t(k) d k,
$$

which shows 1.28. 
Remark 1.7. For given functions $A$ and $B$, it is known that a general solution to the Liouville equation (1.4) with $H=0$ is represented as

$$
\omega(u, v)=\left(\int_{0}^{u} \phi(k) d k-\int_{0}^{v} \psi(k) d k\right)\left(-\frac{A(u) B(v)}{\phi(u) \psi(v)}\right)^{1 / 2},
$$

where $\phi$ and $\psi$ are arbitrary functions with no zeros in one variable.

Corollary 1.8 (Representation formula). Let $\gamma_{1}, \gamma_{2}$ be plane curves defined on intervals $I_{1}$, $I_{2}$ respectively. Assume that both the intervals contain 0 . Then the map

$$
f(u, v)=\left[\begin{array}{c}
\gamma_{1}(u)+\gamma_{2}(v) \\
z(u, v)
\end{array}\right]
$$

where the height $z$ is defined by

$$
z(u, v)=\operatorname{det}\left[\gamma_{1}(u), \gamma_{2}(v)\right]+\int_{0}^{u} \operatorname{det}\left[\gamma_{1}(k), \gamma_{1}^{\prime}(k)\right] d k-\int_{0}^{v} \operatorname{det}\left[\gamma_{2}(k), \gamma_{2}^{\prime}(k)\right] d k
$$

is an indefinite improper affine sphere with the affine normal ${ }^{\mathrm{t}}[0,0,1]$, which is parametrized by the asymptotic coordinates $(u, v) \in \mathbb{D}=I_{1} \times I_{2}$. Its affine metric $h=2 \omega d u d v$ and cubic form $C=A d u^{3}+B d v^{3}$ are given by

$$
\omega=\operatorname{det}\left[\gamma_{1}^{\prime}(u), \gamma_{2}^{\prime}(v)\right], \quad A=\operatorname{det}\left[\gamma_{1}^{\prime}(u), \gamma_{1}^{\prime \prime}(u)\right], \quad B=\operatorname{det}\left[\gamma_{2}^{\prime \prime}(v), \gamma_{2}^{\prime}(v)\right] .
$$

The singular set of $f$ is $S=\left\{(u, v) \in \mathbb{D} \mid \operatorname{det}\left[\gamma_{1}^{\prime}(u), \gamma_{2}^{\prime}(v)\right]=0\right\}$. Moreover the associated family of $f$ is given by the transformation

$$
\gamma_{1} \mapsto\left[\begin{array}{cc}
\lambda & 0 \\
0 & \lambda^{2}
\end{array}\right] \gamma_{1}, \quad \gamma_{2} \mapsto\left[\begin{array}{cc}
\lambda^{-2} & 0 \\
0 & \lambda^{-1}
\end{array}\right] \gamma_{2}
$$

where $\lambda \in \mathbb{R}^{\times}$. Conversely all indefinite improper affine spheres can be locally constructed in this way.

Proof. First, introducing functions $p=a b-c$ and $q=r s-t$, we rephrase (1.28) as

$$
f^{\lambda}=\left[\begin{array}{c}
\lambda a+\lambda^{-2} q \\
\lambda^{2} p+\lambda^{-1} r \\
a r-p q+\lambda^{3} \int_{0}^{u}\left(a p^{\prime}-a^{\prime} p\right) d k-\lambda^{-3} \int_{0}^{v}\left(q r^{\prime}-q^{\prime} r\right) d k
\end{array}\right]
$$

where we use the identities

$$
\begin{aligned}
\alpha c & =a^{\prime} c+a\left(a b^{\prime}-c^{\prime}\right) \\
& =a^{2} b^{\prime}-a c^{\prime}+a^{\prime} c \\
& =a p^{\prime}-a^{\prime} p,
\end{aligned}
$$

and $\rho t=q^{\prime} r-q r^{\prime}$. We note that $a(0)=p(0)=q(0)=r(0)=0$. We then consider an equiaffine transformation of $f^{\lambda}$ as

$$
\tilde{f}^{\lambda}=\left[\begin{array}{ccc}
1 & 0 & 0 \\
0 & 1 & 0 \\
\lambda^{-1} r_{0}-\lambda^{2} p_{0} & \lambda a_{0}-\lambda^{-2} q_{0} & 1
\end{array}\right] f^{\lambda}+\left[\begin{array}{c}
\lambda a_{0}+\lambda^{-2} q_{0} \\
\lambda^{2} p_{0}+\lambda^{-1} r_{0} \\
a_{0} r_{0}-p_{0} q_{0}
\end{array}\right],
$$

where $a_{0}, r_{0}, p_{0}$ and $q_{0}$ are some constants. A straightforward computation shows that

$$
\tilde{f}^{\lambda}=\left[\begin{array}{c}
\lambda \tilde{a}+\lambda^{-2} \tilde{q} \\
\lambda^{2} \tilde{p}+\lambda^{-1} \tilde{r} \\
\tilde{a} \tilde{r}-\tilde{p} \tilde{q}+\lambda^{3} \int_{0}^{u}\left(\tilde{a} \tilde{p}^{\prime}-\tilde{a}^{\prime} \tilde{p}\right) d k-\lambda^{-3} \int_{0}^{v}\left(\tilde{q} \tilde{r}^{\prime}-\tilde{q}^{\prime} \tilde{r}\right) d k
\end{array}\right],
$$


where $\tilde{a}=a+a_{0}, \tilde{p}=p+p_{0}, \tilde{q}=q+q_{0}$ and $\tilde{r}=r+r_{0}$. Thus we obtain 1.30 on writing

$$
\gamma_{1}(u)=\left[\begin{array}{l}
\tilde{a}(u) \\
\tilde{p}(u)
\end{array}\right], \quad \gamma_{2}(v)=\left[\begin{array}{c}
\tilde{q}(v) \\
\tilde{r}(v)
\end{array}\right] .
$$

Since $\gamma_{1}$ and $\gamma_{2}$ are arbitrary, (1.30) gives the all improper indefinite affine spheres.

The formula (1.32) is exactly the same that is represented in [1, p. 216]. In contrast to the Blaschke's proof which utilized the Lelieuvre's formula, our proof is based on the decomposition of the extended frame.

Remark 1.9. The representation formula (1.30) is also formulated in [6] with their concern in computer vision. They have given a geometric interpretation of the height function (1.31) as follows. Consider the curves $2 \gamma_{1}$ and $2 \gamma_{2}$, and fix two points $2 \gamma_{1}(u)$ and $2 \gamma_{2}(v)$ arbitrarily. We assume both $u$ and $v$ are positive for simplicity, and denote by $\Omega$ the region enclosed by the union of four curves

$$
\begin{aligned}
& C_{1}:[0, v] \ni k \mapsto 2 \gamma_{2}(k), \\
& C_{2}:[0,1] \ni k \mapsto 2 \gamma_{2}(v)+k\left(2 \gamma_{1}(u)-2 \gamma_{2}(v)\right), \\
& C_{3}:[0, u] \ni k \mapsto 2 \gamma_{1}(-k+u), \\
& C_{4}:[0,1] \ni k \mapsto 2 \gamma_{1}(0)+k\left(2 \gamma_{2}(0)-2 \gamma_{1}(0)\right) .
\end{aligned}
$$

Then the value $|z(u, v)|$ gives the area of the region $\Omega$. We will again mention this fact in a simplified case, see Example 2 .

We illustrate some examples of indefinite improper affine spheres by using the representation formula (1.30). The resulting surfaces usually have singularities, and hence are sometimes called indefinite improper affine maps, which were introduced in [18] for non-convex improper affine surfaces as an analogue of convex ones [15].

Example 1. Let $P$ and $R$ be smooth functions in one variable. We substitute graphs

$$
\gamma_{1}(u)=\left[\begin{array}{c}
u \\
P^{\prime}(u)
\end{array}\right], \quad \gamma_{2}(v)=\left[\begin{array}{c}
R^{\prime}(v) \\
v
\end{array}\right]
$$

into the representation formula (1.30), and have an indefinite improper affine sphere

$$
f(u, v)=\left[\begin{array}{c}
u+R^{\prime}(v) \\
v+P^{\prime}(u) \\
\left(u+R^{\prime}(v)\right)\left(v+P^{\prime}(u)\right)-2\left(P(u)+R(v)+P^{\prime}(u) R^{\prime}(v)\right)
\end{array}\right] .
$$

Its data is $\omega=1-P^{\prime \prime}(u) R^{\prime \prime}(v), A=P^{\prime \prime \prime}(u), B=R^{\prime \prime \prime}(v)$. It describes a subclass of indefinite improper affine spheres that may have singularities. In view of singularity theory it is known that a cuspidal cross cap, which is one of the typical singularities as well as cuspidal edges or swallowtails, never appear on indefinite improper affine spheres. See [18] and [11] for details.

Especially we set $R=0$ so that we have a smooth indefinite improper affine sphere

$$
f(u, v)=\left[\begin{array}{c}
u \\
w \\
u w-2 P(u)
\end{array}\right],
$$

where $w=v+P^{\prime}(u)$. Further, the most simplest choice $P=0$ gives the hyperbolic paraboloid, or the choice $P(u)=(1 / 6) u^{3}$ gives the Cayley surface. It is known that if the affine metric of an indefinite improper affine sphere is flat then it is locally of the form (1.34). 
Example 2. If $\gamma_{1}$ is the same as $\gamma_{2}$, we write them as $\gamma$, the formula (1.30) becomes

$$
\begin{gathered}
f(u, v)=\left[\begin{array}{c}
\gamma(u)+\gamma(v) \\
z(u, v)
\end{array}\right], \\
z(u, v)=\operatorname{det}[\gamma(u), \gamma(v)]+\int_{v}^{u} \operatorname{det}\left[\gamma(k), \gamma^{\prime}(k)\right] d k .
\end{gathered}
$$

It has the data

$$
\omega=\left|\operatorname{det}\left[\gamma^{\prime}(u), \gamma^{\prime}(v)\right]\right|, \quad A=\operatorname{det}\left[\gamma^{\prime}(u), \gamma^{\prime \prime}(u)\right], \quad B=-\operatorname{det}\left[\gamma^{\prime}(v), \gamma^{\prime \prime}(v)\right] .
$$

A geometric interpretation of the function (1.36) is given in [5], which is called the inner area distance in their language. Here we briefly explain what it is. Consider the curve $2 \gamma$, and fix two points $G_{0}$ and $G_{1}$ on its image arbitrarily. We write

$$
G_{0}=2 \gamma(u)=2\left[\begin{array}{l}
a(u) \\
p(u)
\end{array}\right], \quad G_{1}=2 \gamma(v)=2\left[\begin{array}{l}
a(v) \\
p(v)
\end{array}\right]
$$

and assume $u<v$ for simplicity. We denote by $\Omega$ the region bounded by the union of two curves, the $\operatorname{arc} C_{1}:[u, v] \ni k \mapsto 2 \gamma(k)$ and the line segment $C_{2}:[0,1] \ni k \mapsto G_{1}+k\left(G_{0}-G_{1}\right)$. Then, by the Green's theorem, the area of $\Omega \subset\left(\mathbb{R}^{2},(x, y)\right)$ is computed by the line integral

$$
\begin{aligned}
\frac{1}{2} \int_{C_{1}+C_{2}}-y d x+x d y= & \int_{u}^{v}\left(-p(k) a^{\prime}(k)+a(k) p^{\prime}(k)\right) d k \\
& +\int_{0}^{1}-(p(v)+k(p(u)-p(v)))(a(u)-a(v)) d k \\
& +\int_{0}^{1}(a(v)+k(a(u)-a(v)))(p(u)-p(v)) d k \\
= & -z(u, v) .
\end{aligned}
$$

Namely, the representation formula (1.35 says that, at the midpoint of the line segment connecting $2 \gamma(u)$ and $2 \gamma(v)$, the height $z(u, v)$ is given by the signed area of the region $\Omega$. We also note that it is found in [5] that, when $\gamma$ is closed, we can introduce new variables $x$, $y$ and $\psi$ by the graph expression of (1.35) as

$$
\left[\begin{array}{c}
x \\
y \\
\psi(x, y)
\end{array}\right]=\left[\begin{array}{c}
\gamma(u)+\gamma(v) \\
z(u, v)
\end{array}\right]
$$

so as to obtain a solution to the Monge-Ampère equation with the Dirichlet boundary condition

$$
\left(\partial_{x}^{2} \psi\right)\left(\partial_{y}^{2} \psi\right)-\left(\partial_{x} \partial_{y} \psi\right)^{2}=-1,\left.\quad \psi\right|_{\partial \Gamma}=0
$$

where $\Gamma$ denotes the region bounded by the closed curve $2 \gamma$. We can readily verify (1.37) by a direct computation as follows. From the definition of new variables we have that

$$
\begin{aligned}
& \partial_{u} \psi=\operatorname{det}\left[\gamma(u)-\gamma(v), \gamma^{\prime}(u)\right], \\
& \partial_{v} \psi=\operatorname{det}\left[\gamma(u)-\gamma(v), \gamma^{\prime}(v)\right],
\end{aligned}
$$

and the differential relation $\left[\partial_{u}, \partial_{v}\right]=\left[\partial_{x}, \partial_{y}\right]\left[\gamma^{\prime}(u), \gamma^{\prime}(v)\right]$. This implies that

$$
\left[\begin{array}{c}
\partial_{y} \psi \\
\partial_{x} \psi
\end{array}\right]=\left[\begin{array}{cc}
1 & 0 \\
0 & -1
\end{array}\right](\gamma(u)-\gamma(v))
$$


and hence we have the Hesse matrix of $\psi$ as

$$
\left[\partial_{y}\left[\begin{array}{c}
\partial_{y} \psi \\
\partial_{x} \psi
\end{array}\right], \partial_{x}\left[\begin{array}{c}
\partial_{y} \psi \\
\partial_{x} \psi
\end{array}\right]\right]=\frac{1}{\operatorname{det} M(u, v)} M(u, v){ }^{\mathrm{t}} M(v, u)
$$

where

$$
M(u, v)=\left[\begin{array}{cc}
1 & 0 \\
0 & -1
\end{array}\right]\left[\gamma^{\prime}(u), \gamma^{\prime}(v)\right]
$$

Therefore the determinant of Hesse matrix of $\psi$ is identically -1 because $\operatorname{det}^{\mathrm{t}} M(v, u)=$ $-\operatorname{det} M(u, v)$. Thus the formula (1.35) also provides us with a construction method of solutions to (1.37). Now we illustrate some examples by taking several closed curves $\gamma$.

(1) First one is given by the circle

$$
\gamma(u)=\left[\begin{array}{c}
\cos u \\
\sin u
\end{array}\right]
$$

which leads to

$$
f(u, v)=\left[\begin{array}{c}
\cos u+\cos v \\
\sin u+\sin v \\
u-v-\sin (u-v)
\end{array}\right]=2\left[\begin{array}{c}
\cos x \cos y \\
\cos x \sin y \\
x-\cos x \sin x
\end{array}\right]
$$

where $x=(u-v) / 2$ and $y=(u+v) / 2$. Its data is

$$
\omega=\sin (u-v), \quad A=1, \quad B=-1 .
$$

Therefore $f$ has singularities at $S=\left\{(u, v) \in \mathbb{R}^{2} \mid u \equiv v(\bmod \pi)\right\}$.

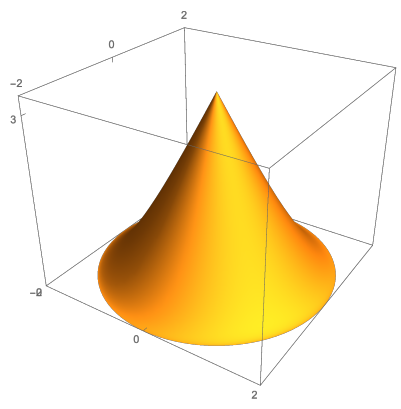

Figure 1. An indefinite improper affine sphere $f(0<x<\pi / 2,-\pi \leq y<\pi)$ over the region enclosed by $2 \gamma$. 

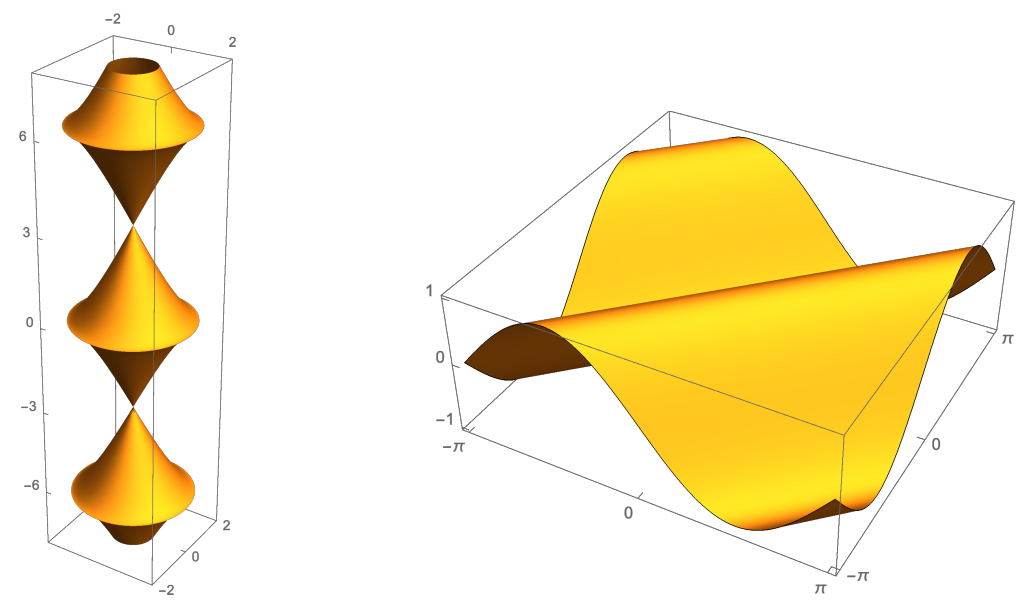

FiguRE 2. Left: an indefinite improper affine map $f$, which is a series of surfaces in Figure 1, joined along cuspidal edges and at cone points. Right: the graph of $\omega$, which gives the affine metric of $f$ apart from $S$.

(2) Second example is given by the square

$$
\gamma(u)=\left[\begin{array}{c}
|\cos u| \cos u \\
|\sin u| \sin u
\end{array}\right] \text {. }
$$

We have that $\operatorname{det}\left[\gamma(u), \gamma^{\prime}(u)\right]=|\sin 2 u|$ for $u \in \mathbb{R} \backslash(\pi / 2) \mathbb{Z}$, which follows from

$$
\gamma^{\prime}(u)=2\left[\begin{array}{c}
-|\cos u| \sin u \\
|\sin u| \cos u
\end{array}\right], \quad \gamma^{\prime \prime}(u)=2 \cos 2 u\left[\begin{array}{c}
-\operatorname{sign}(\cos u) \\
\operatorname{sign}(\sin u)
\end{array}\right],
$$

where

$$
\operatorname{sign} x= \begin{cases}1 & (x>0) \\ 0 & (x=0) \\ -1 & (x<0) .\end{cases}
$$

It is convenient for the following discussion to interpret $\gamma^{\prime}(k)=0$ and $\gamma^{\prime \prime}(k) \| \gamma(k)$ for all $k \in(\pi / 2) \mathbb{Z}$. It holds for all $u \in \mathbb{R}$ that

$$
\int_{0}^{u}|\sin 2 k| d k=\left\lceil\frac{2}{\pi} u\right\rceil-\frac{\operatorname{sign}(\sin 2 u)}{2}(\cos 2 u+\operatorname{sign}(\sin 2 u)),
$$

where we denote by $\lceil u\rceil$ the ceiling of $u$, that is, the smallest integer greater than or equal to $u$. Thus we have for $u, v \in \mathbb{R} \backslash(\pi / 2) \mathbb{Z}$ that

$$
f(u, v)=\left[\begin{array}{c}
|\cos u| \cos u+|\cos v| \cos v \\
|\sin u| \sin u+|\sin v| \sin v \\
z(u, v)
\end{array}\right],
$$

where

$$
\begin{aligned}
z(u, v)= & |\cos u \sin v| \cos u \sin v-|\cos v \sin u| \cos v \sin u \\
& +\lceil(2 / \pi) u\rceil-(1 / 2) \operatorname{sign}(\sin 2 u)(\cos 2 u+\operatorname{sign}(\sin 2 u)) \\
& -\lceil(2 / \pi) v\rceil+(1 / 2) \operatorname{sign}(\sin 2 v)(\cos 2 v+\operatorname{sign}(\sin 2 v)) .
\end{aligned}
$$

Its data is $A=B=0$ and

$$
\omega=4(-|\cos u \sin v| \cos v \sin u+|\cos v \sin u| \cos u \sin v) \text {. }
$$


The singular set $S$ is a checkerboard

$$
S=\left\{(u, v) \in \mathbb{R}^{2} \mid u \in \frac{\pi}{2} \mathbb{Z} \text { or } v \in \frac{\pi}{2} \mathbb{Z} \text { or }\left\lceil\frac{2}{\pi} u\right\rceil \equiv\left\lceil\frac{2}{\pi} v\right\rceil(\bmod 2)\right\} .
$$
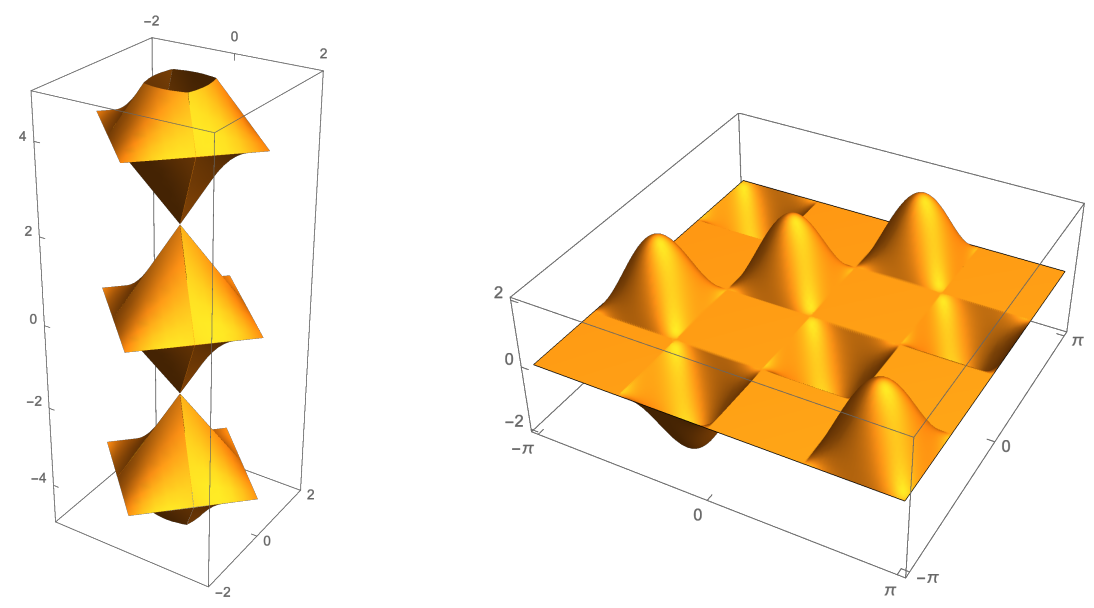

Figure 3. An indefinite improper affine map $f$ (left), and its affine metric $\omega$ (right).

(3) Last example is given by the curve

$$
\gamma(u)=\cos u\left[\begin{array}{c}
1 / 2+\cos ^{2} u \\
2 \sin u
\end{array}\right] .
$$

We have $\operatorname{det}\left[\gamma(u), \gamma^{\prime}(u)\right]=\left(3+2 \sin ^{2} u\right) \cos ^{3} u$ and hence

$$
\int_{0}^{u} \operatorname{det}\left[\gamma(k), \gamma^{\prime}(k)\right] d k=\frac{5}{2} \sin u+\frac{5}{24} \sin 3 u-\frac{1}{40} \sin 5 u \text {. }
$$

Therefore

$$
f(u, v)=\left[\begin{array}{c}
(1+(1 / 2) \cos 2 u) \cos u+(1+(1 / 2) \cos 2 v) \cos v \\
\sin 2 u+\sin 2 v \\
z(u, v)
\end{array}\right]
$$

where

$$
\begin{aligned}
z(u, v)= & -\cos u \cos v(\sin u-\sin v)(3+2 \sin u \sin v) \\
& +\frac{5}{2}(\sin u-\sin v)+\frac{5}{24}(\sin 3 u-\sin 3 v)-\frac{1}{40}(\sin 5 u-\sin 5 v) .
\end{aligned}
$$

Its data is

$$
\begin{aligned}
\omega & =-(\sin u-\sin v)(4+8 \sin u \sin v+3 \cos 2 u \cos 2 v), \\
A & =\frac{1}{2}(19-8 \cos 2 u+3 \cos 4 u) \cos u, \\
B & =-\frac{1}{2}(19-8 \cos 2 v+3 \cos 4 v) \cos v .
\end{aligned}
$$

The singular set of $f$ is $S=S_{1} \cup S_{2}$, where

$$
\begin{aligned}
& S_{1}=\left\{(u, v) \in \mathbb{R}^{2} \mid v \equiv u(\bmod 2 \pi), v \equiv-u+\pi(\bmod 2 \pi)\right\}, \\
& S_{2}=\left\{(u, v) \in \mathbb{R}^{2} \mid 4+8 \sin u \sin v+3 \cos 2 u \cos 2 v=0\right\} .
\end{aligned}
$$


The sets $S_{1}$ and $S_{2}$ consist of lines and circlelike curves, respectively. The surface is compact and of genus 1 .
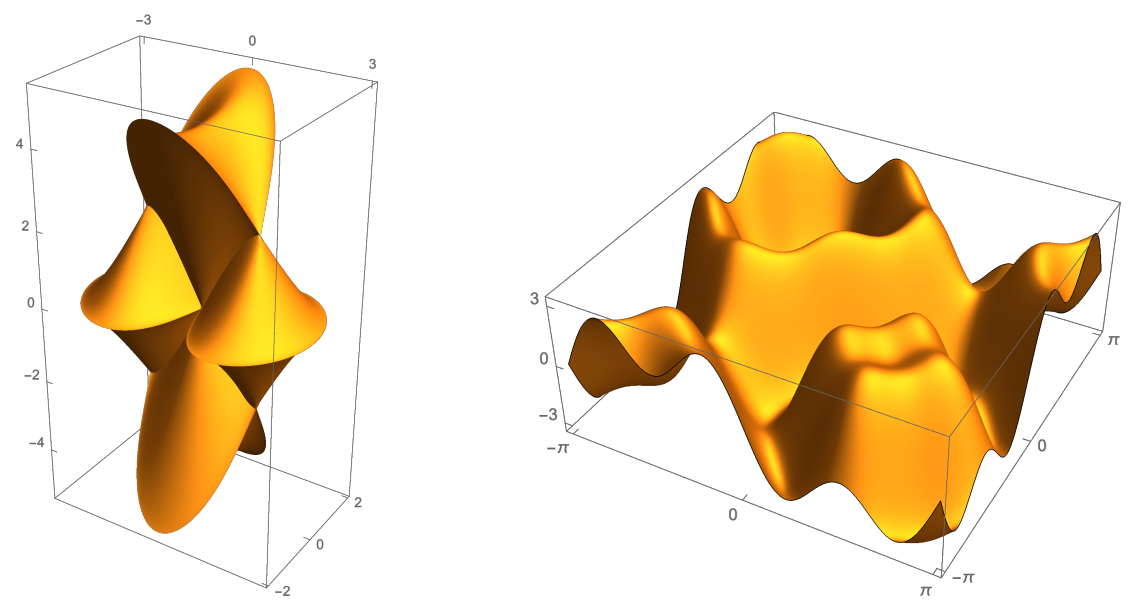

Figure 4. An indefinite improper affine map $f$ (left), and its affine metric $\omega$ (right).

\section{Discrete indefinite AFFine SPHERES}

In the previous section we derived Theorem 1.3 and Corollary 1.8 which offer a Weierstrass type representation formula of indefinite affine spheres. Based on a technique of decompositions of the loop group, we shall generalize this formula to discrete case, and obtain a Weierstrass type representation formula for discrete indefinite affine spheres.

2.1. Definitions. Let $f: \mathbb{Z}^{2} \rightarrow \mathbb{R}^{3},(n, m) \mapsto f_{n}^{m}$ be a map. We call $f$ a discrete indefinite affine sphere if it satisfies the following two properties ([3], [2], [16]):

(1) Every five points $f_{n}^{m}, f_{n \pm 1}^{m}, f_{n}^{m \pm 1}$ lie on a plane.

(2) The line $\ell_{n}^{m}$ connecting two points $f_{n+1}^{m+1}+f_{n}^{m}$ and $f_{n+1}^{m}+f_{n}^{m+1}$ satisfies either of the following two conditions:

(a) All the lines $\ell_{n}^{m}$ meet at one point in $\mathbb{R}^{3}$.

(b) All the lines $\ell_{n}^{m}$ are parallel to each other.

A discrete indefinite affine sphere $f$ is said to be proper if it satisfies the condition (2a), or improper if (2b).

If $f$ is a discrete indefinite affine sphere, the vector $f_{n+1}^{m+1}-f_{n+1}^{m}-f_{n}^{m+1}+f_{n}^{m}$ is parallel to the discrete affine normal

$$
\xi_{n}^{m}=-H \frac{f_{n+1}^{m}+f_{n}^{m+1}}{2}+(1+H) \xi_{0},
$$

where $\xi_{0}$ is a constant vector. Here we set $H=-1$ if $f$ is proper, and $H=0$ if $f$ is improper. Without loss of generality we can fix $\xi_{0}$ to be ${ }^{\mathrm{t}}[0,0,1]$. Taking into account a continuum limit, we introduce positive numbers $\epsilon$ and $\delta$, which play a role of lattice intervals. In view of this it may be better to regard $f$ as a map $f: \epsilon \mathbb{Z} \times \delta \mathbb{Z} \rightarrow \mathbb{R}^{3}$, and hence entries of $f$ depend on $\epsilon$ and $\delta$. We define

$$
\tilde{F}_{n}^{m}=\left[\frac{f_{n+1}^{m}-f_{n}^{m}}{\epsilon}, \frac{f_{n}^{m+1}-f_{n}^{m}}{\delta}, \xi_{n}^{m}\right]
$$


We suppose that $\operatorname{det} \tilde{F}_{n}^{m} \neq 0$, then there exist functions $\omega, A, B$ such that

$$
\begin{aligned}
& \frac{f_{n+1}^{m}-2 f_{n}^{m}+f_{n-1}^{m}}{\epsilon^{2}}=\left(\frac{\omega_{n}^{m}-\omega_{n-1}^{m}}{\epsilon \omega_{n}^{m}}+\frac{\delta H}{2} \omega_{n-1}^{m}\right) \frac{f_{n+1}^{m}-f_{n}^{m}}{\epsilon}+\frac{A_{n}^{m}}{\omega_{n}^{m}} \frac{f_{n}^{m+1}-f_{n}^{m}}{\delta}, \\
& \frac{f_{n+1}^{m+1}-f_{n+1}^{m}-f_{n}^{m+1}+f_{n}^{m}}{\epsilon \delta}=\omega_{n}^{m} \xi_{n}^{m}, \\
& \frac{f_{n}^{m+1}-2 f_{n}^{m}+f_{n}^{m-1}}{\delta^{2}}=\frac{B_{n}^{m}}{\omega_{n}^{m}} \frac{f_{n+1}^{m}-f_{n}^{m}}{\epsilon}+\left(\frac{\omega_{n}^{m}-\omega_{n}^{m-1}}{\delta \omega_{n}^{m}}+\frac{\epsilon H}{2} \omega_{n}^{m-1}\right) \frac{f_{n}^{m+1}-f_{n}^{m}}{\delta} .
\end{aligned}
$$

Equations (2.2) and (2.4) are consequences of the property (1). See [3, p. 118] or [16, Proposition 3.4] for a proof. Throughout the paper we further impose on $f$ the volume condition

$$
\operatorname{det} \tilde{F}_{n}^{m}=\frac{2 \omega_{n}^{m}}{2-\epsilon \delta H \omega_{n}^{m}}
$$

which can be regarded as a discrete analogue of (1.1). We write

$$
g_{n}^{m}=\frac{2}{2-\epsilon \delta H \omega_{n}^{m}}
$$

to have expressions $\omega_{n}^{m} g_{n}^{m}=\operatorname{det} \tilde{F}_{n}^{m}$ and

$$
\begin{aligned}
A_{n}^{m} g_{n}^{m} & =\operatorname{det}\left[\frac{f_{n+1}^{m}-f_{n}^{m}}{\epsilon}, \frac{f_{n+1}^{m}-2 f_{n}^{m}+f_{n-1}^{m}}{\epsilon^{2}}, \xi_{n}^{m}\right], \\
B_{n}^{m} g_{n}^{m} & =\operatorname{det}\left[\frac{f_{n}^{m+1}-2 f_{n}^{m}+f_{n}^{m-1}}{\delta^{2}}, \frac{f_{n}^{m+1}-f_{n}^{m}}{\delta}, \xi_{n}^{m}\right] .
\end{aligned}
$$

From the compatibility condition among $(2.2)-(2.4)$, it follows that $\omega, A, B$ satisfy the system

$$
\begin{gathered}
\omega_{n+1}^{m+1} \omega_{n}^{m}-\frac{\omega_{n+1}^{m} \omega_{n}^{m+1}}{g_{n+1}^{m+1} g_{n}^{m}}+\epsilon \delta A_{n+1}^{m} B_{n+1}^{m+1}=0, \\
g_{n+1}^{m+1} A_{n+1}^{m+1}=g_{n}^{m} A_{n+1}^{m}, \quad g_{n+1}^{m+1} B_{n+1}^{m+1}=g_{n}^{m} B_{n}^{m+1} .
\end{gathered}
$$

Indeed, the matrix $\tilde{F}_{n}^{m}$ varies according to the system

$$
\tilde{F}_{n+1}^{m}=\tilde{F}_{n}^{m} \tilde{U}_{n}^{m}, \quad \tilde{F}_{n}^{m+1}=\tilde{F}_{n}^{m} \tilde{V}_{n}^{m},
$$

where coefficient matrices are computed as

$$
\begin{gathered}
\tilde{U}_{n}^{m}=\left[\begin{array}{ccc}
\frac{\omega_{n+1}^{m}}{\omega_{m}^{m}} g_{n+1}^{m} & 0 & -\epsilon \frac{H}{2}\left(1+\frac{\omega_{n+1}^{m}}{\omega_{n}^{m}} g_{n+1}^{m}\right) \\
\epsilon \frac{A_{n+1}^{m}}{\omega_{n}^{m}} g_{n+1}^{m} & 1 & -\epsilon^{2} \frac{H}{2} \frac{A_{n+1}^{m}}{\omega_{n}^{m}} g_{n+1}^{m} \\
\epsilon^{2} A_{n+1}^{m} g_{n+1}^{m} & \epsilon \omega_{n}^{m} & \frac{1}{g_{n}^{m}}-\epsilon^{3} \frac{H}{2} A_{n+1}^{m} g_{n+1}^{m}
\end{array}\right], \\
\tilde{V}_{n}^{m}=\left[\begin{array}{ccc}
1 & \delta \frac{B_{n}^{m+1}}{\omega_{n}^{m}} g_{n}^{m+1} & -\delta^{2} \frac{H}{2} \frac{B_{n}^{m+1}}{\omega_{n}^{m}} g_{n}^{m+1} \\
0 & \frac{\omega_{n}^{m+1}}{\omega_{n}^{m}} g_{n}^{m+1} & -\delta \frac{H}{2}\left(1+\frac{\omega_{n}^{m+1}}{\omega_{n}^{m}} g_{n}^{m+1}\right) \\
\delta \omega_{n}^{m} & \delta^{2} B_{n}^{m+1} g_{n}^{m+1} & \frac{1}{g_{n}^{m}}-\delta^{3} \frac{H}{2} B_{n}^{m+1} g_{n}^{m+1}
\end{array}\right] .
\end{gathered}
$$

The compatibility condition $\tilde{U}_{n}^{m} \tilde{V}_{n+1}^{m}=\tilde{V}_{n}^{m} \tilde{U}_{n}^{m+1}$ is (2.6)-2.7). Therefore the system (2.8)(2.10), or equivalently (2.2)-(2.4), has a solution if and only if $(2.6)-(2.7)$ hold. The system (2.6)-(2.7) is a discrete analogue of the system (1.4)-(1.5), and hence called the discrete Tzitzeica equation if $H=-1$, or the discrete Liouville equation if $H=0$. Since this system (2.6) - 2.7) is invariant under the transformation

$$
A_{n}^{m} \mapsto \lambda^{3} A_{n}^{m}, \quad B_{n}^{m} \mapsto \lambda^{-3} B_{n}^{m}, \quad \lambda \in \mathbb{R}^{\times},
$$


the discrete affine sphere $f$ has 1-parameter family, which we call the associated family of $f$. The associated family preserves $\omega$.

2.2. Loop group description. In order to derive a representation formula for discrete indefinite affine spheres, we use decomposition techniques of loop groups. To begin with, following [3], we describe the discrete indefinite affine spheres in terms of the loop groups. We set

$$
F_{n}^{m}=\tilde{F}_{n}^{m}\left[\begin{array}{ccc}
\lambda^{-1} & 0 & \epsilon H / 2 \\
0 & \lambda\left(\omega_{n}^{m} g_{n}^{m}\right)^{-1} & \delta H / 2 \\
0 & 0 & 1
\end{array}\right] .
$$

Then the map $F$ is $\mathrm{SL}_{3} \mathbb{R}$-valued, and satisfies the system

$$
F_{n+1}^{m}=F_{n}^{m} U_{n}^{m}, \quad F_{n}^{m+1}=F_{n}^{m} V_{n}^{m},
$$

where $U$ and $V$ are computed as

$$
\begin{aligned}
U_{n}^{m} & =\left[\begin{array}{ccc}
\frac{\omega_{n+1}^{m} g_{n+1}^{m}}{\omega_{n}^{m}}-\frac{H}{2} A_{n+1}^{m} g_{n+1}^{m}(\epsilon \lambda)^{3} & -\frac{H}{2} \frac{\omega_{n}^{m}}{\omega_{n+1}^{m} g_{n+1}^{m}}(\epsilon \lambda)^{2} & -H \epsilon \lambda \\
A_{n+1}^{m} g_{n+1}^{m} \epsilon \lambda & \frac{\omega_{n}^{m}}{\omega_{n+1}^{m} g_{n+1}^{m}} & 0 \\
A_{n+1}^{m} g_{n+1}^{m}(\epsilon \lambda)^{2} & \frac{\omega_{n}^{m}}{\omega_{n+1}^{m} g_{n+1}^{m}} \epsilon \lambda & 1
\end{array}\right], \\
V_{n}^{m} & =\left[\begin{array}{ccc}
\frac{1}{g_{n}^{m}} & \frac{B_{n}^{m+1}}{\omega_{n}^{m+1} \omega_{m}^{m} g_{n}^{m}} \delta \lambda^{-1} & 0 \\
-\frac{H}{2}\left(\omega_{n}^{m}\right)^{2} g_{n}^{m}\left(\delta \lambda^{-1}\right)^{2} & g_{n}^{m}-\frac{H}{2} \frac{B_{n}^{m+1} \omega_{n}^{m} g_{n}^{m}}{\omega_{n}^{m+1}}\left(\delta \lambda^{-1}\right)^{3} & -H \omega_{n}^{m} g_{n}^{m} \delta \lambda^{-1} \\
\omega_{n}^{m} \delta \lambda^{-1} & \frac{B_{n}^{m+1}}{\omega_{n}^{m+1}}\left(\delta \lambda^{-1}\right)^{2} & 1
\end{array}\right] .
\end{aligned}
$$

The consistency of (2.12), that is, $U_{n}^{m} V_{n+1}^{m}=V_{n}^{m} U_{n}^{m+1}$, is of course given by (2.6)-(2.7). By multiplying $F$ by some constant matrix from the left if necessary, without loss of generality we can assume that

$$
F_{0}^{0}=\mathrm{id}
$$

at the base point $(n, m)=(0,0)$. The family of gauged frames $F$ defined by (2.11) with the initial condition (2.15) will be called the extended frame of discrete affine sphere $f$. The extended frame $F$ is obviously a $\Lambda \mathrm{SL}_{3} \mathbb{R}$-valued map. Conversely, if the matrices $\bar{U}_{n}^{m}$ and $\bar{V}_{n}^{m}$ have similar entries as (2.13) and (2.14) respectively, then they give the extended family of discrete indefinite affine spheres. In fact we have the following proposition, which has been shown for the discrete indefinite proper affine spheres $(H=-1)$ in [3, Theorem in Section 6].

Proposition 2.1. Let $\bar{U}_{n}^{m}$ and $\bar{V}_{n}^{m}$ be matrices which depend on a parameter $\lambda \in \mathbb{R}^{\times}$as

$$
\begin{aligned}
& \bar{U}_{n}^{m}=\bar{U}_{n, m}^{0}+\lambda \bar{U}_{n, m}^{1}+\lambda^{2} \bar{U}_{n, m}^{2}+\lambda^{3} \bar{U}_{n, m}^{3}, \\
& \bar{V}_{n}^{m}=\bar{V}_{n, m}^{0}+\lambda^{-1} \bar{V}_{n, m}^{1}+\lambda^{-2} \bar{V}_{n, m}^{2}+\lambda^{-3} \bar{V}_{n, m}^{3} .
\end{aligned}
$$

Here coefficient matrices $\bar{U}^{i}$ and $\bar{V}^{i}$, which are labeled by the index $i(0 \leq i \leq 3)$, have the entries

$$
\begin{gathered}
\bar{U}_{n, m}^{0}=\operatorname{diag}\left(1 / u_{n, m}^{22}, u_{n, m}^{22}, 1\right), \quad \bar{U}_{n, m}^{3}=\operatorname{diag}\left(-(H / 2)\left(u_{n, m}^{13}\right)^{2} u_{n, m}^{21}, 0,0\right), \\
\bar{U}_{n, m}^{1}=\left[\begin{array}{ccc}
0 & 0 & -H u_{n, m}^{13} \\
u_{n, m}^{21} & 0 & 0 \\
0 & u_{n, m}^{22} u_{n, m}^{13} & 0
\end{array}\right], \quad \bar{U}_{n, m}^{2}=\left[\begin{array}{ccc}
0 & -\frac{H}{2} u_{n, m}^{22}\left(u_{n, m}^{13}\right)^{2} & 0 \\
0 & 0 & 0 \\
u_{n, m}^{13} u_{n, m}^{21} & 0 & 0
\end{array}\right],
\end{gathered}
$$


and

$$
\begin{aligned}
& \bar{V}_{n, m}^{0}=\operatorname{diag}\left(v_{n, m}^{11}, 1 / v_{n, m}^{11}, 1\right), \quad \bar{V}_{n, m}^{3}=\operatorname{diag}\left(0,-(H / 2)\left(v_{n, m}^{23}\right)^{2} v_{n, m}^{12}, 0\right), \\
& \bar{V}_{n, m}^{1}=\left[\begin{array}{ccc}
0 & v_{n, m}^{12} & 0 \\
0 & 0 & -H v_{n, m}^{23} \\
v_{n, m}^{11} v_{n, m}^{23} & 0 & 0
\end{array}\right], \quad \bar{V}_{n, m}^{2}=\left[\begin{array}{ccc}
0 & 0 & 0 \\
-\frac{H}{2} v_{n, m}^{11}\left(v_{n, m}^{23}\right)^{2} & 0 & 0 \\
0 & v_{n, m}^{23} v_{n, m}^{12} & 0
\end{array}\right] \text {, }
\end{aligned}
$$

with some functions $u^{21}, v^{12}$, and nowhere vanishing functions $u^{13}, u^{22}, v^{11}, v^{23}$, and a constant $H \in\{-1,0\}$. If $\bar{U}$ and $\bar{V}$ satisfy the relation $\bar{U}_{n}^{m} \bar{V}_{n+1}^{m}=\bar{V}_{n}^{m} \bar{U}_{n}^{m+1}$ for all $\lambda$, then there exist a map $\bar{F}$ and a gauge $D$ such that

(1) $\bar{F}$ satisfies the system $\bar{F}_{n+1}^{m}=\bar{F}_{n}^{m} \bar{U}_{n}^{m}, \bar{F}_{n}^{m+1}=\bar{F}_{n}^{m} \bar{V}_{n}^{m}$ and

(2) $\left(D_{0}^{0}\right)^{-1} \bar{F}_{n}^{m} D_{n}^{m}$ is the extended frame of some discrete indefinite affine sphere.

Proof. Because of the relation $\bar{U}_{n}^{m} \bar{V}_{n+1}^{m}=\bar{V}_{n}^{m} \bar{U}_{n}^{m+1}$, the existence of $\bar{F}$ is clear. We fix a positive number $\epsilon$, and set

$$
F_{n}^{m}=\bar{F}_{n}^{m} D_{n}^{m}, \quad D_{n}^{m}=\operatorname{diag}\left(u_{n, m}^{13} / \epsilon, \epsilon / u_{n, m}^{13}, 1\right) .
$$

Then $F$ satisfies $F_{n+1}^{m}=F_{n}^{m} U_{n}^{m}$ and $F_{n}^{m+1}=F_{n}^{m} V_{n}^{m}$, where

$$
\begin{aligned}
& U_{n}^{m}=\left(D_{n}^{m}\right)^{-1} \bar{U}_{n}^{m} D_{n+1}^{m} \\
& =\left[\begin{array}{ccc}
\frac{u_{n+1, m}^{13}}{u_{n, m}^{13} u_{n, m}^{22}}-\lambda^{3} \frac{H}{2} u_{n, m}^{13} u_{n, m}^{21} u_{n+1, m}^{13} & -\frac{H}{2} \lambda^{2} \frac{\epsilon^{2} u_{n, m}^{13} u_{n, m}^{22}}{u_{n}^{2}+1, m} & -H \epsilon \lambda \\
\lambda \frac{u_{n, m}^{13} u_{n, m}^{21} u_{n+1, m}^{13}}{\epsilon^{2}} & \frac{u_{n, m}^{13} u_{n, m}^{22}}{u_{n+1}^{13}} & 0 \\
\lambda^{2} \frac{u_{n, m}^{13} u_{n, m}^{21} u_{n+1, m}^{13}}{\epsilon} & \lambda \frac{\epsilon n_{n, m}^{13} u_{n, m}^{22}}{u_{n+1, m}^{13}} & 1
\end{array}\right], \\
& V_{n}^{m}=\left(D_{n}^{m}\right)^{-1} \bar{V}_{n}^{m} D_{n}^{m+1} \\
& =\left[\begin{array}{ccc}
\frac{v_{n, m}^{11} u_{n, m+1}^{13}}{u_{n, m}^{13}} & \frac{\epsilon^{2} v_{n, m}^{12}}{\lambda u_{n, m}^{13} u_{n, m+1}^{13}} & 0 \\
-\frac{H}{2} \frac{u_{n, m}^{13} u_{n, m+1}^{13} v_{n, m}^{11}\left(v_{n, m}^{23}\right)^{2}}{\lambda^{2} \epsilon^{2}} & \frac{u_{n, m}^{13}}{u_{n, m+1}^{13} v_{n, m}^{11}}-\frac{H}{2} \frac{u_{n, m}^{13} v_{n, m}^{12}\left(v_{n, m}^{23}\right)^{2}}{\lambda^{3} u_{n, m+1}^{13}} & -H \frac{u_{n, m}^{13} v_{n, m}^{23}}{\lambda \epsilon} \\
\frac{v_{n, m}^{11} v_{n, m}^{23} u_{n, m+1}^{13}}{\lambda \epsilon} & \frac{\epsilon v_{n, m}^{12} v_{n, m}^{23}}{\lambda^{2} u_{n, m+1}^{13}} & 1
\end{array}\right] .
\end{aligned}
$$

Next we fix a positive number $\delta$ and introduce sequences $\omega, A, B$ by

$$
\omega_{n}^{m}=\frac{v_{n, m}^{11} v_{n, m}^{23} u_{n, m+1}^{13}}{\epsilon \delta},
$$

and

$$
A_{n}^{m}=\frac{u_{n-1, m}^{13} u_{n-1, m}^{21} u_{n, m}^{13}}{\epsilon^{3} g_{n}^{m}}, \quad B_{n}^{m}=\frac{\epsilon v_{n, m-1}^{12} v_{n, m-1}^{22}}{\delta^{2} u_{n, m}^{13}} \omega_{n}^{m},
$$

where $g$ is defined by 2.5). Therefore the matrices $U_{n}^{m}$ and $V_{n}^{m}$ are written as

$$
\begin{aligned}
U_{n}^{m} & =\left[\begin{array}{ccc}
\frac{1}{r_{n}^{m}}-\lambda^{3} \epsilon^{3} \frac{H}{2} A_{n+1}^{m} g_{n+1}^{m} & -\frac{H}{2} \lambda^{2} \epsilon^{2} r_{n}^{m} & -H \epsilon \lambda \\
\lambda \epsilon A_{n+1}^{m} g_{n+1}^{m} & r_{n}^{m} & 0 \\
\lambda^{2} \epsilon^{2} A_{n+1}^{m} g_{n+1}^{m} & \lambda \epsilon r_{n}^{m} & 1
\end{array}\right], \\
V_{n}^{m} & =\left[\begin{array}{ccc}
\frac{1}{h_{n}^{m}} & \frac{\delta B_{n}^{m+1}}{\lambda \omega_{n}^{m+1} h_{m}^{m}} & 0 \\
-\frac{H}{2} \frac{\delta^{2}\left(\omega_{n}^{m}\right)^{2} h_{n}^{m}}{\lambda^{2}} & h_{n}^{m}-\frac{H}{2} \frac{\delta^{3} B_{n}^{m+1} \omega_{n}^{m} h_{n}^{m}}{\lambda^{3} \omega_{n}^{m+1}} & -H \frac{\delta h_{n}^{m} \omega_{n}^{m}}{\lambda} \\
\frac{\delta \omega_{n}^{m}}{\lambda} & \frac{\delta^{2} B_{n}^{m+1}}{\lambda^{2} \omega_{n}^{m+1}} & 1
\end{array}\right],
\end{aligned}
$$


where

$$
h_{n}^{m}=\frac{u_{n, m}^{13}}{u_{n, m+1}^{13} v_{n, m}^{11}}, \quad r_{n}^{m}=\frac{u_{n, m}^{13} u_{n, m}^{22}}{u_{n+1, m}^{13}} .
$$

We note that the condition $\bar{U}_{n}^{m} \bar{V}_{n+1}^{m}=\bar{V}_{n}^{m} \bar{U}_{n}^{m+1}$ is equivalent to

$$
U_{n}^{m} V_{n+1}^{m}=V_{n}^{m} U_{n}^{m+1},
$$

which implies that

$$
h_{n}^{m}=g_{n}^{m}, \quad r_{n}^{m}=\frac{\omega_{n}^{m}}{\omega_{n+1}^{m} g_{n+1}^{m}} .
$$

In fact, comparing the $(2,2)$ - and $(3,2)$-entries of the both sides of 2.17$)$, we have

$$
\begin{aligned}
& h_{n+1}^{m} r_{n}^{m}+\frac{\epsilon \delta g_{n+1}^{m} A_{n+1}^{m} B_{n+1}^{m+1}}{h_{n+1}^{m} \omega_{n+1}^{m} \omega_{n+1}^{m+1}}=\frac{h_{n}^{m} r_{n}^{m+1}}{\left(g_{n}^{m}\right)^{2}}, \\
& h_{n+1}^{m} r_{n}^{m}+\frac{\epsilon \delta g_{n+1}^{m} A_{n+1}^{m} B_{n+1}^{m+1}}{h_{n+1}^{m} \omega_{n+1}^{m} \omega_{n+1}^{m+1}}=\frac{r_{n}^{m+1}}{g_{n}^{m}} .
\end{aligned}
$$

Then it immediately follows that $h$ should be $g$. Further, from $(1,2)$ - and $(3,2)$-entries of (2.17), we have

$$
\begin{aligned}
& \frac{g_{n+1}^{m} B_{n+1}^{m+1}\left(2-\epsilon \delta H\left(1+r_{n}^{m}\right) \omega_{n+1}^{m}\right) \omega_{n}^{m+1}}{2 \omega_{n+1}^{m+1} B_{n}^{m+1} r_{n}^{m+1}}=\frac{2 r_{n}^{m} \omega_{n+1}^{m}}{g_{n}^{m} \omega_{n}^{m}\left(2-\epsilon \delta H\left(1+r_{n}^{m}\right) \omega_{n+1}^{m}\right)}, \\
& \frac{g_{n+1}^{m} B_{n+1}^{m+1}\left(2-\epsilon \delta H\left(1+r_{n}^{m}\right) \omega_{n+1}^{m}\right) \omega_{n}^{m+1}}{2 \omega_{n+1}^{m+1} B_{n}^{m+1} r_{n}^{m+1}}=1,
\end{aligned}
$$

which implies that $r_{n}^{m} \omega_{n+1}^{m} g_{n+1}^{m}=\omega_{n}^{m}$. Thus $U_{n}^{m}$ and $V_{n}^{m}$ become exactly the same as 2.13) and 2.14). We conclude that $\left(D_{0}^{0}\right)^{-1} F_{n}^{m}$ is the extended frame of some discrete indefinite affine sphere.

We are now in position to state one of the main theorems of this paper, which is a discrete analogue of Theorem 1.3 .

Theorem 2.2. Let $\epsilon$ and $\delta$ be positive numbers, and $F: \mathbb{Z}^{2} \rightarrow \Lambda \mathrm{SL}_{3} \mathbb{R}$ be the extended frame of a discrete indefinite affine sphere with the discrete affine normal (2.1). By the Birkhoff decomposition, we decompose $F_{n}^{m}$ near $(n, m)=(0,0)$ as

$$
F_{n}^{m}=F_{n, m}^{+} F_{n, m}^{-}=G_{n, m}^{-} G_{n, m}^{+},
$$

where $F_{n, m}^{+} \in \Lambda_{*}^{+} \mathrm{SL}_{3} \mathbb{R}, F_{n, m}^{-} \in \Lambda^{-} \mathrm{SL}_{3} \mathbb{R}, G_{n, m}^{-} \in \Lambda_{*}^{-} \mathrm{SL}_{3} \mathbb{R}$ and $G_{n, m}^{+} \in \Lambda^{+} \mathrm{SL}_{3} \mathbb{R}$. Then $F^{+}$ and $G^{-}$do not depend on $m$ and $n$, respectively, that is, they satisfy that

$$
F_{n, m+1}^{+}=F_{n, m}^{+}, \quad G_{n+1, m}^{-}=G_{n, m}^{-} .
$$

We write $F_{n}^{+}$for $F_{n, m}^{+}$and $G_{m}^{-}$for $G_{n, m}^{-}$, so that we have the ordinary difference equations

$$
F_{n+1}^{+}=F_{n}^{+} \xi_{n}^{+}, \quad G_{m+1}^{-}=G_{m}^{-} \xi_{m}^{-}
$$


with

$$
\begin{aligned}
& \xi_{n}^{+}=\left[\begin{array}{ccc}
1-\frac{H}{2}\left(\alpha_{n+1}\right)^{2} \beta_{n+1}(\epsilon \lambda)^{3} & -\frac{H}{2}\left(\alpha_{n+1}\right)^{2}(\epsilon \lambda)^{2} & -H \alpha_{n+1} \epsilon \lambda \\
\beta_{n+1} \epsilon \lambda & 1 & 0 \\
\alpha_{n+1} \beta_{n+1}(\epsilon \lambda)^{2} & \alpha_{n+1} \epsilon \lambda & 1
\end{array}\right] \\
& \xi_{m}^{-}=\left[\begin{array}{ccc}
1 & \sigma_{m+1} \delta \lambda^{-1} & 0 \\
-\frac{H}{2}\left(\rho_{m+1}\right)^{2}\left(\delta \lambda^{-1}\right)^{2} & 1-\frac{H}{2} \sigma_{m+1}\left(\rho_{m+1}\right)^{2}\left(\delta \lambda^{-1}\right)^{3} & -H \rho_{m+1} \delta \lambda^{-1} \\
\rho_{m+1} \delta \lambda^{-1} & \sigma_{m+1} \rho_{m+1}\left(\delta \lambda^{-1}\right)^{2} & 1
\end{array}\right],
\end{aligned}
$$

where functions $\alpha, \beta$ depend only on $n$, and $\sigma, \rho$ only on $m$. Moreover $\alpha_{n} \neq 0$ and $\rho_{m} \neq 0$ for all $n$ and $m$.

Conversely, let $\alpha_{n}, \beta_{n}$ be functions depending only on $n$, and $\sigma_{m}, \rho_{m}$ functions depending only on $m$. Assume that $\alpha_{n}$ and $\rho_{m}$ have no zeros. Let $F_{n}^{+}$and $G_{m}^{-}$be solutions to the system (2.19) - 2.21) with the initial condition $F_{0}^{+}=G_{0}^{-}=\mathrm{id}$. Define $V_{n, m}^{+} \in \Lambda_{*}^{+} \mathrm{SL}_{3} \mathbb{R}$ and $V_{n, m}^{-} \in \Lambda^{-} \mathrm{SL}_{3} \mathbb{R}$ by the Birkhoff decomposition for $\left(G_{m}^{-}\right)^{-1} F_{n}^{+}$near $(n, m)=(0,0)$ as

$$
\left(G_{m}^{-}\right)^{-1} F_{n}^{+}=V_{n, m}^{+}\left(V_{n, m}^{-}\right)^{-1}
$$

and write $\hat{F}_{n}^{m}=F_{n}^{+} V_{n, m}^{-}=G_{m}^{-} V_{n, m}^{+}$. Then there exists a diagonal matrix $D_{n}^{m}$ such that $\left(D_{0}^{0}\right)^{-1} \hat{F}_{n}^{m} D_{n}^{m}$ is the extended frame of a discrete indefinite affine sphere $f_{n}^{m}$. In particular, in case of discrete indefinite proper affine spheres $(H=-1)$, the third column of the extended frame $\left(D_{0}^{0}\right)^{-1} \hat{F}_{n}^{m} D_{n}^{m}$ directly gives the position vector of $f_{n}^{m}$.

Proof. Let $F$ be an extended frame, and define $F^{+}$and $F^{-}$by $(2.18)$. Therefore we have $F_{n, m}^{+}=F_{n, m}\left(F_{n, m}^{-}\right)^{-1}$ and so that

$$
\left(F_{n, m}^{+}\right)^{-1} F_{n, m+1}^{+}=F_{n, m}^{-}\left(F_{n}^{m}\right)^{-1} F_{n}^{m+1}\left(F_{n, m+1}^{-}\right)^{-1}=F_{n, m}^{-} V_{n}^{m}\left(F_{n, m+1}^{-}\right)^{-1},
$$

where $V_{n}^{m}$ is given by (2.14). The left-hand side takes values in $\Lambda_{*}^{+} \mathrm{SL}_{3} \mathbb{R}$ and the right-hand side takes values in $\Lambda^{-} \mathrm{SL}_{3} \mathbb{R}$. Thus $\left(F_{n, m}^{+}\right)^{-1} F_{n, m+1}^{+}=\mathrm{id}$. Similarly $\left(G_{n, m}^{-}\right)^{-1} G_{n+1, m}^{-}$is identity matrix. Therefore $F^{+}$and $G^{-}$do not depend on $m$ and $n$, respectively.

Next, let us compute $\left(F_{n}^{+}\right)^{-1} F_{n+1}^{+}$and $\left(G_{m}^{-}\right)^{-1} G_{m+1}^{-}$. It is straightforward to see that

$$
\left(F_{n}^{+}\right)^{-1} F_{n+1}^{+}=F_{n, m}^{-}\left(F_{n}^{m}\right)^{-1} F_{n+1}^{m}\left(F_{n+1, m}^{-}\right)^{-1}=F_{n, m}^{-} U_{n}^{m}\left(F_{n+1, m}^{-}\right)^{-1}
$$

where $U_{n}^{m}$ is given by 2.13. Since $U_{n}^{m}$ has the form $U_{n}^{m}=\sum_{k=0}^{3} \lambda^{k} U_{n, m}^{k}$ and $F_{n, m}^{-}$takes values in $\Lambda^{-} \mathrm{SL}_{3} \mathbb{R}$, we have

$$
\xi_{n}^{+}=\left(F_{n}^{+}\right)^{-1} F_{n+1}^{+}=X_{n}^{0}+\lambda X_{n}^{1}+\lambda^{2} X_{n}^{2}+\lambda^{3} X_{n}^{3} .
$$

With the expansions

$$
\begin{aligned}
F_{n, m}^{-} & =I_{n, m}^{0}+\lambda^{-1} I_{n, m}^{1}+\lambda^{-2} I_{n, m}^{2}+\lambda^{-3} I_{n, m}^{3}+\cdots, \\
\left(F_{n, m}^{-}\right)^{-1} & =J_{n, m}^{0}+\lambda^{-1} J_{n, m}^{1}+\lambda^{-2} J_{n, m}^{2}+\lambda^{-3} J_{n, m}^{3}+\cdots,
\end{aligned}
$$


it is easy to see that $X_{n}^{0}, X_{n}^{1}, X_{n}^{2}, X_{n}^{3}$ are computed as

$$
\begin{aligned}
X_{n}^{0}= & I_{n, m}^{0} U_{n, m}^{3} J_{n+1, m}^{3}+I_{n, m}^{1} U_{n, m}^{3} J_{n+1, m}^{2}+I_{n, m}^{2} U_{n, m}^{3} J_{n+1, m}^{1}+I_{n, m}^{3} U_{n, m}^{3} J_{n+1, m}^{0} \\
& +I_{n, m}^{0} U_{n, m}^{2} J_{n+1, m}^{2}+I_{n, m}^{1} U_{n, m}^{2} J_{n+1, m}^{1}+I_{n, m}^{2} U_{n, m}^{2} J_{n+1, m}^{0} \\
& +I_{n, m}^{0} U_{n, m}^{1} J_{n+1, m}^{1}+I_{n, m}^{1} U_{n, m}^{1} J_{n+1, m}^{0}+I_{n, m}^{0} U_{n, m}^{0} J_{n+1, m}^{0}, \\
X_{n}^{1}= & I_{n, m}^{0} U_{n, m}^{3} J_{n+1, m}^{2}+I_{n, m}^{1} U_{n, m}^{3} J_{n+1, m}^{1}+I_{n, m}^{2} U_{n, m}^{3} J_{n+1, m}^{0} \\
& +I_{n, m}^{0} U_{n, m}^{2} J_{n+1, m}^{1}+I_{n, m}^{1} U_{n, m}^{2} J_{n+1, m}^{0}+I_{n, m}^{0} U_{n, m}^{1} J_{n+1, m}^{0}, \\
X_{n}^{2}= & I_{n, m}^{0} U_{n, m}^{3} J_{n+1, m}^{1}+I_{n, m}^{1} U_{n, m}^{3} J_{n+1, m}^{0}+I_{n, m}^{0} U_{n, m}^{2} J_{n+1, m}^{0}, \\
X_{n}^{3}= & I_{n, m}^{0} U_{n, m}^{3} J_{n+1, m}^{0} .
\end{aligned}
$$

From Proposition 1.1, every one of $\left\{I_{n, m}^{0}, J_{n, m}^{0}, I_{n, m}^{3}, J_{n, m}^{3}\right\},\left\{I_{n, m}^{1}, J_{n, m}^{1}\right\},\left\{I_{n, m}^{2}, J_{n, m}^{2}\right\}$ has the following form

$$
\operatorname{diag}(*, *, *), \quad\left[\begin{array}{ccc}
0 & * & 0 \\
0 & 0 & * \\
* & 0 & 0
\end{array}\right], \quad\left[\begin{array}{lll}
0 & 0 & * \\
* & 0 & 0 \\
0 & * & 0
\end{array}\right],
$$

respectively. Since $\xi_{n}^{+}$takes values in $\Lambda_{*}^{+} \mathrm{SL} \mathrm{L}_{3} \mathbb{R}$, thus $X_{n}^{0}=\mathrm{id}$. Noticing that $U_{n, m}^{1}, U_{n, m}^{2}, U_{n, m}^{3}$ are given by (2.13), it readily follows that the coefficients $X_{n}^{k}$ have the form

$$
X_{n}^{3}=\operatorname{diag}\left(x_{n}^{11}, 0,0\right), \quad X_{n}^{1}=\left[\begin{array}{ccc}
0 & 0 & x_{n}^{13} \\
x_{n}^{21} & 0 & 0 \\
0 & x_{n}^{32} & 0
\end{array}\right], \quad X_{n}^{2}=\left[\begin{array}{ccc}
0 & x_{n}^{12} & 0 \\
0 & 0 & 0 \\
x_{n}^{31} & 0 & 0
\end{array}\right],
$$

where $x_{n}^{i j}$ are some functions in $n$. Thus we have

$$
\xi_{n}^{+}=\left[\begin{array}{ccc}
1+\lambda^{3} x_{n}^{11} & \lambda^{2} x_{n}^{12} & \lambda x_{n}^{13} \\
\lambda x_{n}^{21} & 1 & 0 \\
\lambda^{2} x^{31} & \lambda x_{n}^{32} & 1
\end{array}\right] .
$$

We now consider the twisted condition 1.12 , namely ${ }^{t} \xi(-\lambda) T \xi(\lambda)=T$. It is easy to see that the twisted condition is equivalent to the system

$$
x_{n}^{13}=-H x_{n}^{32}, \quad x_{n}^{31}=x_{n}^{21} x_{n}^{32}, \quad x_{n}^{12}=-\frac{H}{2}\left(x_{n}^{32}\right)^{2}, \quad x_{n}^{11}=-\frac{H}{2}\left(x_{n}^{32}\right)^{2} x_{n}^{21} .
$$

Thus we have

$$
\xi_{n}^{+}=\left[\begin{array}{ccc}
1-\lambda^{3} \frac{H}{2}\left(x_{n}^{32}\right)^{2} x_{n}^{21} & -\lambda^{2} \frac{H}{2}\left(x_{n}^{32}\right)^{2} & -\lambda H x_{n}^{32} \\
\lambda x_{n}^{21} & 1 & 0 \\
\lambda^{2} x_{n}^{21} x_{n}^{32} & \lambda x_{n}^{32} & 1
\end{array}\right]
$$

and the expression 2.20 on rewriting $x_{n}^{32}=\epsilon \alpha_{n+1}$ and $x_{n}^{21}=\epsilon \beta_{n+1}$. We write $P(i, j)$ for the $(i, j)$-entry of a matrix $P$, and show that $\alpha_{n+1}$ has no zeros as follows. We compute $x_{n}^{32}$, $x_{n}^{12}$ and $X_{n}^{0}(2,2)$ so that we have

$$
\begin{aligned}
x_{n}^{32} & =\epsilon\left(-2+\epsilon H I_{n, m}^{1}(3,1)\right) C_{n}^{m}, \\
x_{n}^{12} & =\epsilon^{2} H I_{n, m}^{0}(1,1) C_{n}^{m}, \\
X_{n}^{0}(2,2) & =\left(-\frac{2}{I_{n, m}^{0}(1,1)}+\epsilon^{2} H I_{n, m}^{2}(2,1)-2 \epsilon I_{n, m}^{2}(2,3)\right) C_{n}^{m},
\end{aligned}
$$

where we set

$$
a_{n}^{m}=\frac{\omega_{n+1}^{m} g_{n+1}^{m}}{\omega_{n}^{m}} \neq 0, \quad b_{n}^{m}=A_{n+1}^{m} g_{n+1}^{m}, \quad C_{n}^{m}=-\frac{1+a_{n}^{m} b_{n}^{m} J_{n+1, m}^{0}(1,1) J_{n+1, m}^{1}(1,2)}{2 a_{n}^{m} J_{n+1, m}^{0}(1,1)} .
$$


Because of the condition $X_{n}^{0}(2,2)=1$, expressions 2.23$)$ and $(2.25)$ imply that $x_{n}^{32}$ has no zeros if $H=0$. When $H=-1$, expressions (2.24) and 2.25) imply $x_{n}^{32} \neq 0$ because $x_{n}^{12}=-(H / 2)\left(x_{n}^{32}\right)^{2}$. Similarly, $\left(G_{m}^{-}\right)^{-1} G_{m+1}^{-}$can be computed as in 2.21) with a nowhere vanishing function $\rho_{m}$.

Conversely, let $\left(F_{n}^{+}, G_{m}^{-}\right)$be a pair of solutions of $(2.19)$ such that $F_{0}^{+}=G_{0}^{-}=$id. We write

$$
\xi_{n}^{+}=\sum_{j=0}^{3} \lambda^{j} X_{n}^{j}, \quad \xi_{m}^{-}=\sum_{j=0}^{3} \lambda^{-j} Y_{m}^{j}
$$

where coefficient matrices $X^{j}$ and $Y^{j}$ are defined by (2.20) and (2.21). Consider the Birkhoff decomposition of $\left(G_{m}^{-}\right)^{-1} F_{n}^{+}$near $(n, m)=(0,0)$ as $\left(G_{m}^{-}\right)^{-1} F_{n}^{+}=V_{n, m}^{+}\left(V_{n, m}^{-}\right)^{-1}$ and define $\hat{F}_{n}^{m}=F_{n}^{+} V_{n, m}^{-}=G_{m}^{-} V_{n, m}^{+}$. We express

$$
V_{n, m}^{-}=\sum_{j=0}^{\infty} \lambda^{-j} K_{n, m}^{j}, \quad V_{n, m}^{+}=\sum_{j=0}^{\infty} \lambda^{j} M_{n, m}^{j} .
$$

Their inverses are

$$
\left(V_{n, m}^{-}\right)^{-1}=\sum_{j=0}^{\infty} \lambda^{-j} L_{n, m}^{j}, \quad\left(V_{n, m}^{+}\right)^{-1}=\sum_{j=0}^{\infty} \lambda^{j} N_{n, m}^{j}
$$

with $K_{n, m}^{0} L_{n, m}^{0}=$ id and $M_{n, m}^{0}=N_{n, m}^{0}=\mathrm{id}$, and for all $j \geq 0$ it holds that

$$
\sum_{k=0}^{j+1} K_{n, m}^{k} L_{n, m}^{j+1-k}=0, \quad \sum_{k=0}^{j+1} M_{n, m}^{k} N_{n, m}^{j+1-k}=0 .
$$

Namely the matrices $L^{j+1}$ and $N^{j+1}$ are computed as

$$
\begin{aligned}
& L_{n, m}^{1}=-L_{n, m}^{0} K_{n, m}^{1} L_{n, m}^{0}, \\
& L_{n, m}^{2}=-L_{n, m}^{0}\left(K_{n, m}^{2}-K_{n, m}^{1} L_{n, m}^{0} K_{n, m}^{1}\right) L_{n, m}^{0}, \\
& L_{n, m}^{3}=-L_{n, m}^{0}\left(K_{n, m}^{3}-K_{n, m}^{1} L_{n, m}^{0} K_{n, m}^{2}-K_{n, m}^{2} L_{n, m}^{0} K_{n, m}^{1}\right. \\
& \left.\quad \quad+K_{n, m}^{1} L_{n, m}^{0} K_{n, m}^{1} L_{n, m}^{0} K_{n, m}^{1}\right) L_{n, m}^{0}, \\
& N_{n, m}^{1}=-M_{n, m}^{1}, \\
& N_{n, m}^{2}=-M_{n, m}^{2}+\left(M_{n, m}^{1}\right)^{2}, \\
& N_{n, m}^{3}=-M_{n, m}^{3}+M_{n, m}^{1} M_{n, m}^{2}+M_{n, m}^{2} M_{n, m}^{1}-\left(M_{n, m}^{1}\right)^{3},
\end{aligned}
$$

and so forth. Further, from the twisted condition 1.12 , it holds that

$$
(-1)^{j \mathrm{t}} L_{n, m}^{j} T=T K_{n, m}^{j}, \quad(-1)^{j \mathrm{t}} N_{n, m}^{j} T=T M_{n, m}^{j}
$$

for all $j \geq 0$. In particular, setting $j=0$, we have that

$$
K_{n, m}^{0}=\operatorname{diag}\left(k_{n}^{m}, 1 / k_{n}^{m}, 1\right), \quad L_{n, m}^{0}=\left(K_{n, m}^{0}\right)^{-1}
$$


where $k$ is some sequence which has no zeros. For higher $j$, we have that

$$
\begin{aligned}
& K_{n, m}^{1}=\left[\begin{array}{ccc}
0 & \kappa_{n, m}^{12} & 0 \\
0 & 0 & -\frac{H}{k_{n}^{m}} \kappa_{n, m}^{31} \\
\kappa_{n, m}^{31} & 0 & 0
\end{array}\right] \\
& K_{n, m}^{2}=\left[\begin{array}{ccc}
0 & 0 & H\left(k_{n}^{m} \kappa_{n, m}^{32}-\kappa_{n, m}^{12} \kappa_{n, m}^{31}\right) \\
-\frac{H}{2 k_{n}^{m}}\left(\kappa_{n, m}^{31}\right)^{2} & 0 & 0 \\
0 & \kappa_{n, m}^{32} & 0
\end{array}\right] \text {, } \\
& K_{n, m}^{3}=\operatorname{diag}\left(\left(k_{n}^{m}\right)^{2} \kappa_{n, m}^{22}+\frac{H}{2} \kappa_{n, m}^{31}\left(2 k_{n}^{m} \kappa_{n, m}^{32}-\kappa_{n, m}^{12} \kappa_{n, m}^{31}\right), \kappa_{n, m}^{22}, \kappa_{n, m}^{33}\right) \text {, } \\
& M_{n, m}^{1}=\left[\begin{array}{ccc}
0 & 0 & -H \mu_{n, m}^{32} \\
\mu_{n, m}^{21} & 0 & 0 \\
0 & \mu_{n, m}^{32} & 0
\end{array}\right] \text {, } \\
& M_{n, m}^{2}=\left[\begin{array}{ccc}
0 & -\frac{H}{2}\left(\mu_{n, m}^{32}\right)^{2} & 0 \\
0 & 0 & H\left(\mu_{n, m}^{31}-\mu_{n, m}^{21} \mu_{n, m}^{32}\right) \\
\mu_{n, m}^{31} & 0 & 0
\end{array}\right] \text {, } \\
& M_{n, m}^{3}=\operatorname{diag}\left(\mu_{n, m}^{11}, \mu_{n, m}^{11}+\frac{H}{2} \mu_{n, m}^{32}\left(2 \mu_{n, m}^{31}-\mu_{n, m}^{21} \mu_{n, m}^{32}\right), \mu_{n, m}^{33}\right) \text {, }
\end{aligned}
$$

and so on. Here $\kappa^{i j}$ and $\mu^{i j}$ are some sequences in $n, m$. Now we are ready to compute the Maurer-Cartan form of $\hat{F}$. As for $\left(\hat{F}_{n}^{m}\right)^{-1} \hat{F}_{n+1}^{m}$, we have

$$
\begin{aligned}
& \left(\hat{F}_{n}^{m}\right)^{-1} \hat{F}_{n+1}^{m}=\left(V_{n, m}^{+}\right)^{-1} V_{n+1, m}^{+}, \\
& \left(\hat{F}_{n}^{m}\right)^{-1} \hat{F}_{n+1}^{m}=\left(V_{n, m}^{-}\right)^{-1} \xi_{n}^{+} V_{n+1, m}^{-}
\end{aligned}
$$

Comparing these two expressions, it readily follows that there exist matrices such that

$$
\left(\hat{F}_{n}^{m}\right)^{-1} \hat{F}_{n+1}^{m}=\mathrm{id}+\lambda \hat{U}_{n, m}^{1}+\lambda^{2} \hat{U}_{n, m}^{2}+\lambda^{3} \hat{U}_{n, m}^{3} .
$$

Similarly, from

$$
\begin{aligned}
& \left(\hat{F}_{n}^{m}\right)^{-1} \hat{F}_{n}^{m+1}=\left(V_{n, m}^{-}\right)^{-1} V_{n, m+1}^{-}, \\
& \left(\hat{F}_{n}^{m}\right)^{-1} \hat{F}_{n}^{m+1}=\left(V_{n, m}^{+}\right)^{-1} \xi_{m}^{-} V_{n, m+1}^{+},
\end{aligned}
$$

we have that

$$
\left(\hat{F}_{n}^{m}\right)^{-1} \hat{F}_{n}^{m+1}=\hat{V}_{n, m}^{0}+\lambda^{-1} \hat{V}_{n, m}^{1}+\lambda^{-2} \hat{V}_{n, m}^{2}+\lambda^{-3} \hat{V}_{n, m}^{3} .
$$

From (2.27) and (2.29), it readily follows that the coefficient matrices are of the form

$$
\begin{aligned}
& \hat{U}_{n, m}^{1}=\left[\begin{array}{lll}
0 & 0 & * \\
* & 0 & 0 \\
0 & * & 0
\end{array}\right], \quad \hat{U}_{n, m}^{2}=\left[\begin{array}{ccc}
0 & * & 0 \\
0 & 0 & 0 \\
* & 0 & 0
\end{array}\right], \quad \hat{U}_{n, m}^{3}=\operatorname{diag}(*, 0,0), \\
& \hat{V}_{n, m}^{1}=\left[\begin{array}{ccc}
0 & * & 0 \\
0 & 0 & * \\
* & 0 & 0
\end{array}\right], \quad \hat{V}_{n, m}^{2}=\left[\begin{array}{ccc}
0 & 0 & 0 \\
* & 0 & 0 \\
0 & * & 0
\end{array}\right], \quad \hat{V}_{n, m}^{3}=\operatorname{diag}(0, *, 0) \text {, }
\end{aligned}
$$


and $\hat{V}_{n, m}^{0}$ is diagonal. On the other hand, from 2.26), we have

$$
\begin{aligned}
& \hat{U}_{n, m}^{1}=M_{n+1, m}^{1}+N_{n, m}^{1} \\
& =M_{n+1, m}^{1}-M_{n, m}^{1} \\
& =\left[\begin{array}{ccc}
0 & 0 & -H u_{n, m}^{13} \\
u_{n, m}^{21} & 0 & 0 \\
0 & u_{n, m}^{13} & 0
\end{array}\right], \\
& \hat{U}_{n, m}^{2}=M_{n+1, m}^{2}+N_{n, m}^{1} M_{n+1, m}^{1}+N_{n, m}^{2} \\
& =M_{n+1, m}^{2}-M_{n, m}^{2}-M_{n, m}^{1} \hat{U}_{n, m}^{1} \\
& =\left[\begin{array}{ccc}
0 & -\frac{H}{2}\left(u_{n, m}^{13}\right)^{2} & 0 \\
0 & 0 & H u_{n, m}^{23} \\
u_{n, m}^{31} & 0 & 0
\end{array}\right] \text {, } \\
& \hat{U}_{n, m}^{3}=M_{n+1, m}^{3}+N_{n, m}^{1} M_{n+1, m}^{2}+N_{n, m}^{2} M_{n+1, m}^{1}+N_{n, m}^{3} \\
& =M_{n+1, m}^{3}-M_{n, m}^{3}-M_{n, m}^{1} \hat{U}_{n, m}^{2}-M_{n, m}^{2} \hat{U}_{n, m}^{1} \\
& =\operatorname{diag}\left(u_{n, m}^{11}, u_{n, m}^{22}, *\right) \text {, }
\end{aligned}
$$

where

$$
\begin{aligned}
& u_{n, m}^{13}=\mu_{n+1, m}^{32}-\mu_{n, m}^{32}, \\
& u_{n, m}^{21}=\mu_{n+1, m}^{21}-\mu_{n, m}^{21}, \\
& u_{n, m}^{23}=\mu_{n+1, m}^{31}-\mu_{n, m}^{31}-\mu_{n+1, m}^{32} u_{n, m}^{21}, \\
& u_{n, m}^{31}=\mu_{n+1, m}^{31}-\mu_{n, m}^{31}-\mu_{n, m}^{32} u_{n, m}^{21}, \\
& u_{n, m}^{11}=\mu_{n+1, m}^{11}-\mu_{n, m}^{11}+(H / 2) \mu_{n, m}^{32}\left(\mu_{n+1, m}^{32}+u_{n, m}^{13}\right) u_{n, m}^{21}, \\
& u_{n, m}^{22}=\mu_{n+1, m}^{11}-\mu_{n, m}^{11}+(H / 2) \mu_{n+1, m}^{32}\left(\mu_{n+1, m}^{31}-\mu_{n, m}^{31}+u_{n, m}^{23}\right) .
\end{aligned}
$$

Similarly, from (2.28), we have

$$
\hat{V}_{n, m}^{0}=L_{n, m}^{0} K_{n, m+1}^{0}=\operatorname{diag}\left(\frac{k_{n}^{m+1}}{k_{n}^{m}}, \frac{k_{n}^{m}}{k_{n}^{m+1}}, 1\right),
$$


and hence we have that

$$
\begin{aligned}
& \hat{V}_{n, m}^{1}=L_{n, m}^{0} K_{n, m+1}^{1}+L_{n, m}^{1} K_{n, m+1}^{0} \\
& =L_{n, m}^{0}\left(K_{n, m+1}^{1}-K_{n, m}^{1} \hat{V}_{n, m}^{0}\right) \\
& =\left[\begin{array}{ccc}
0 & v_{n, m}^{12} & 0 \\
0 & 0 & -H v_{n, m}^{23} \\
\frac{k_{n}^{m+1}}{k_{n}^{m}} v_{n, m}^{23} & 0 & 0
\end{array}\right], \\
& \hat{V}_{n, m}^{2}=L_{n, m}^{0} K_{n, m+1}^{2}+L_{n, m}^{1} K_{n, m+1}^{1}+L_{n, m}^{2} K_{n, m+1}^{0} \\
& =L_{n, m}^{0}\left(K_{n, m+1}^{2}-K_{n, m}^{2} \hat{V}_{n, m}^{0}-K_{n, m}^{1} \hat{V}_{n, m}^{1}\right) \\
& =\left[\begin{array}{ccc}
0 & 0 & H v_{n, m}^{13} \\
-\frac{H}{2} \frac{k_{n}^{m+1}}{k_{n}^{m}}\left(v_{n, m}^{23}\right)^{2} & 0 & 0 \\
0 & v_{n, m}^{32} & 0
\end{array}\right] \text {, } \\
& \hat{V}_{n, m}^{3}=L_{n, m}^{0} K_{n, m+1}^{3}+L_{n, m}^{1} K_{n, m+1}^{2}+L_{n, m}^{2} K_{n, m+1}^{1}+L_{n, m}^{3} K_{n, m+1}^{0} \\
& =L_{n, m}^{0}\left(K_{n, m+1}^{3}-K_{n, m}^{3} \hat{V}_{n, m}^{0}-K_{n, m}^{2} \hat{V}_{n, m}^{1}-K_{n, m}^{1} \hat{V}_{n, m}^{2}\right) \\
& =\operatorname{diag}\left(v_{n, m}^{11}, v_{n, m}^{22}, *\right) \text {, }
\end{aligned}
$$

where

$$
\begin{aligned}
v_{n, m}^{12} & =\frac{\kappa_{n, m+1}^{12}}{k_{n}^{m}}-\frac{\kappa_{n, m}^{12}}{k_{n}^{m+1}}, \\
v_{n, m}^{23} & =\frac{k_{n}^{m}}{k_{n}^{m+1}} \kappa_{n, m+1}^{31}-\kappa_{n, m}^{31}, \\
v_{n, m}^{13} & =\frac{k_{n}^{m+1}}{k_{n}^{m}}\left(\kappa_{n, m+1}^{32}-\frac{k_{n}^{m}}{k_{n}^{m+1}} \kappa_{n, m}^{32}\right)-\kappa_{n, m+1}^{31} v_{n, m}^{12}, \\
v_{n, m}^{32} & =\kappa_{n, m+1}^{32}-\frac{k_{n}^{m}}{k_{n}^{m+1}} \kappa_{n, m}^{32}-\kappa_{n, m}^{31} v_{n, m}^{12}, \\
v_{n, m}^{11} & =\frac{k_{n}^{m+1}}{k_{n}^{m}}\left(k_{n}^{m+1} \kappa_{n, m+1}^{22}-k_{n}^{m} \kappa_{n, m}^{22}+\frac{H}{2} \kappa_{n, m+1}^{31}\left(\kappa_{n, m+1}^{32}-\frac{k_{n}^{m}}{k_{n}^{m+1}} \kappa_{n, m}^{32}+\frac{k_{n}^{m}}{k_{n}^{m+1}} v_{n, m}^{13}\right)\right), \\
v_{n, m}^{22} & =\frac{k_{n}^{m}}{k_{n}^{m+1}}\left(k_{n}^{m+1} \kappa_{n, m+1}^{22}-k_{n}^{m} \kappa_{n, m}^{22}\right)+\frac{H}{2} \kappa_{n, m}^{31}\left(\kappa_{n, m+1}^{32}-\frac{k_{n}^{m}}{k_{n}^{m+1}} \kappa_{n, m}^{32}+v_{n, m}^{32}\right) .
\end{aligned}
$$

For $H \neq 0$, comparing 2.32 and 2.30 , we have $u_{n, m}^{23}=0$, which implies $u_{n, m}^{31}=u_{n, m}^{13} u_{n, m}^{21}$. For $H=0$, by using (2.27), we have $u_{n, m}^{31}=u_{n, m}^{13} u_{n, m}^{21}$. Comparing (2.33) and (2.30), we have $u_{n, m}^{22}=0$, which implies

$$
\begin{aligned}
u_{n, m}^{11} & =\frac{H}{2} \mu_{n, m}^{32}\left(\mu_{n+1, m}^{32}+u_{n, m}^{13}\right) u_{n, m}^{21}-\frac{H}{2} \mu_{n+1, m}^{32}\left(\mu_{n+1, m}^{31}-\mu_{n, m}^{31}\right) \\
& =\frac{H}{2}\left(\mu_{n+1, m}^{32}-u_{n, m}^{13}\right)\left(\mu_{n+1, m}^{32}+u_{n, m}^{13}\right) u_{n, m}^{21}-\frac{H}{2}\left(\mu_{n+1, m}^{32}\right)^{2} u_{n, m}^{21} \\
& =-\frac{H}{2}\left(u_{n, m}^{13}\right)^{2} u_{n, m}^{21} .
\end{aligned}
$$

For $H \neq 0$, comparing (2.34) and (2.31), we have $v_{n, m}^{13}=0$, which implies $v_{n, m}^{32}=v_{n, m}^{23} v_{n, m}^{12}$. For $H=0$, by using (2.29), we have $v_{n, m}^{32}=v_{n, m}^{23} v_{n, m}^{12}$. Comparing (2.35) and (2.31), we have 
$v_{n, m}^{11}=0$, which implies

$$
\begin{aligned}
v_{n, m}^{22} & =-\frac{H}{2} \frac{k_{n}^{m}}{k_{n}^{m+1}} \kappa_{n, m+1}^{31}\left(\kappa_{n, m+1}^{32}-\frac{k_{n}^{m}}{k_{n}^{m+1}} \kappa_{n, m}^{32}\right)+\frac{H}{2} \kappa_{n, m}^{31}\left(\kappa_{n, m+1}^{32}-\frac{k_{n}^{m}}{k_{n}^{m+1}} \kappa_{n, m}^{32}+v_{n, m}^{32}\right) \\
& =-\frac{H}{2}\left(\frac{k_{n}^{m}}{k_{n}^{m+1}} \kappa_{n, m+1}^{31}-\kappa_{n, m}^{31}\right)\left(\kappa_{n, m+1}^{32}-\frac{k_{n}^{m}}{k_{n}^{m+1}} \kappa_{n, m}^{32}\right)+\frac{H}{2} \kappa_{n, m}^{31} v_{n, m}^{32} \\
& =-\frac{H}{2} v_{n, m}^{23}\left(v_{n, m}^{32}+\kappa_{n, m}^{31} v_{n, m}^{12}\right)+\frac{H}{2} \kappa_{n, m}^{31} v_{n, m}^{32} \\
& =-\frac{H}{2}\left(v_{n, m}^{23}\right)^{2} v_{n, m}^{12} .
\end{aligned}
$$

Finally, again from 2.27 and 2.29$)$, we have

$$
\begin{aligned}
& \left(u_{n, m}^{13}\right)^{2}=\frac{\epsilon^{2}\left(\alpha_{n+1}\right)^{2}}{k_{n}^{m}}\left(\frac{1}{k_{n+1}^{m}}+\epsilon \beta_{n+1} \kappa_{n+1, m}^{12}\right), \\
& \left(v_{n, m}^{23}\right)^{2}=\frac{k_{n}^{m}}{k_{n}^{m+1}} \delta^{2}\left(\rho_{m+1}\right)^{2}\left(1+\delta \sigma_{m+1} \mu_{n, m+1}^{21}\right) .
\end{aligned}
$$

On the other hand, a straightforward computation shows that $(2,2)$-entry of the constant coefficient with respect to $\lambda$ for $\left(\hat{F}_{n}^{m}\right)^{-1} \hat{F}_{n+1}^{m}$ and $(1,1)$-entry of the constant coefficient with respect to $\lambda$ for $\left(\hat{F}_{n}^{m}\right)^{-1} \hat{F}_{n}^{m+1}$ can be respectively computed as

$$
\begin{gathered}
1=\left(\frac{1}{2}+\frac{\epsilon \alpha H \kappa_{n, m}^{31}}{4 k_{n}^{m}}\right)\left(\frac{1}{k_{n+1}^{m}}+\epsilon \beta_{n+1} \kappa_{n+1, m}^{12}\right), \\
0 \neq \frac{k_{n}^{m+1}}{k_{n}^{m}}=\left(1+\frac{\delta \rho H}{2} \mu_{n, m}^{32}\right)^{2}\left(1+\delta \sigma_{m+1} \mu_{n, m+1}^{21}\right) .
\end{gathered}
$$

Thus $1 / k_{n+1}^{m}+\epsilon \beta_{n+1} \kappa_{n+1, m}^{12}$ and $1+\delta \sigma_{m+1} \mu_{n, m+1}^{21}$ have no zeros near $(0,0)$, which implies that $u_{n, m}^{13}$ and $v_{n, m}^{23}$ never vanish near $(n, m)=(0,0)$.

Thus, coefficient matrices $\hat{U}_{n, m}^{j}, \hat{V}_{n, m}^{j}$ satisfy the assumption of Proposition 2.1. Therefore by Proposition 2.1 there exists a diagonal gauge $D_{n}^{m}$ such that $\left(D_{0}^{0}\right)^{-1} \hat{F}_{n}^{m} D_{n}^{m}$ is the extended frame for some discrete indefinite affine sphere.

Remark 2.3. In case of discrete proper affine spheres $(H=-1)$, the functions $A_{n}^{m}$ and $B_{n}^{m}$ do not have simple expressions among the functions $\alpha_{n}, \beta_{n}, \rho_{m}$ and $\sigma_{m}$. On the other hand, in case of discrete improper affine spheres $(H=0)$, they are simply represented as $(2.44)$ below.

2.3. discrete indefinite improper affine spheres. For the rest of this paper, we shall be concerned only with discrete indefinite improper affine spheres $(H=0)$. Equations in 2.7) are simplified as

$$
A_{n+1}^{m+1}=A_{n+1}^{m}, \quad B_{n+1}^{m+1}=B_{n}^{m+1},
$$

which indicate that $A$ and $B$ depend only on $n$ and $m$, respectively. Hence we write $A_{n}$ for $A_{n}^{m}$ and $B_{m}$ for $B_{n}^{m}$. Thus the discrete Liouville equation 2.6 is written as

$$
\omega_{n+1}^{m+1} \omega_{n}^{m}-\omega_{n+1}^{m} \omega_{n}^{m+1}+\epsilon \delta A_{n+1} B_{m+1}=0 .
$$

We now introduce a notation of summation of a sequence $x$ as

$$
\sum_{k}^{n} x_{k}= \begin{cases}\sum_{k=1}^{n} x_{k} & (n \geq 1) \\ 0 & (n=0) \\ -\sum_{k=n+1}^{0} x_{k} & (n \leq-1) \\ 27 & \end{cases}
$$


It holds for all integers $n$ that

$$
\begin{gathered}
\sum_{k}^{n} x_{k}-\sum_{k}^{n-1} x_{k}=x_{n}, \\
\sum_{k}^{n-1} x_{k}-\sum_{k}^{n} x_{k-1}=-x_{0},
\end{gathered}
$$

and hence $\sum_{k}^{n}\left(x_{k}-x_{k-1}\right)=x_{n}-x_{0}$. In particular we have a formula of summation by parts

$$
\sum_{k}^{n} x_{k}\left(y_{k}-y_{k-1}\right)=-x_{0} y_{0}+x_{n} y_{n}-\sum_{k}^{n}\left(x_{k}-x_{k-1}\right) y_{k-1},
$$

which holds for all integers $n$. Then the ordinary difference equations 2.19) can be explicitly computed as follows.

Lemma 2.4. Let $H=0$. Then the pair of solutions $\left(F_{n}^{+}, G_{m}^{-}\right)$to the system (2.19)-2.21) with the initial condition $F_{0}^{+}=G_{0}^{-}=\mathrm{id}$ is explicitly given by

$$
F_{n}^{+}=\left[\begin{array}{ccc}
1 & 0 & 0 \\
b_{n} \lambda & 1 & 0 \\
c_{n} \lambda^{2} & a_{n} \lambda & 1
\end{array}\right], \quad G_{m}^{-}=\left[\begin{array}{ccc}
1 & s_{m} \lambda^{-1} & 0 \\
0 & 1 & 0 \\
r_{m} \lambda^{-1} & t_{m} \lambda^{-2} & 1
\end{array}\right]
$$

where $a, b, c, r, s, t$ are defined as

$$
\begin{gathered}
a_{n}=\epsilon \sum_{k}^{n} \alpha_{k}, \quad b_{n}=\epsilon \sum_{k}^{n} \beta_{k}, \quad c_{n}=\epsilon \sum_{k}^{n} a_{k} \beta_{k}, \\
r_{m}=\delta \sum_{k}^{m} \rho_{k}, \quad s_{m}=\delta \sum_{k}^{m} \sigma_{k}, \quad t_{m}=\delta \sum_{k}^{m} r_{k} \sigma_{k} .
\end{gathered}
$$

Moreover, assume $1-b_{n} s_{m} \neq 0$, and define $V_{n, m}^{+} \in \Lambda_{*}^{+} \mathrm{SL}_{3} \mathbb{R}$ and $V_{n, m}^{-} \in \Lambda^{-} \mathrm{SL}_{3} \mathbb{R}$ by the Birkhoff decomposition of $\left(G_{m}^{-}\right)^{-1} F_{n}^{+}$as

$$
\left(G_{m}^{-}\right)^{-1} F_{n}^{+}=V_{n, m}^{+}\left(V_{n, m}^{-}\right)^{-1}
$$

Then $V^{ \pm}$are explicitly given as

$$
\begin{aligned}
V_{n, m}^{+}= & {\left[\begin{array}{ccc}
1 & 0 & 0 \\
b_{n}\left(1-b_{n} s_{m}\right)^{-1} \lambda & 1 & 0 \\
c_{n}\left(1-b_{n} s_{m}\right)^{-1} \lambda^{2} & \left(a_{n}-s_{m}\left(a_{n} b_{n}-c_{n}\right)\right) \lambda & 1
\end{array}\right], } \\
V_{n, m}^{-}= & {\left[\begin{array}{ccc}
\left(1-b_{n} s_{m}\right)^{-1} & s_{m} \lambda^{-1} & 0 \\
0 & 1-b_{n} s_{m} & 0 \\
\left(r_{m}+b_{n} t_{m}\left(1-b_{n} s_{m}\right)^{-1}\right) \lambda^{-1} & t_{m} \lambda^{-2} & 1
\end{array}\right] . }
\end{aligned}
$$

Proof. It is easy to check that matrices (2.38) satisfy the system $2.19-2.21$ with $H=0$ and $F_{0}^{+}=G_{0}^{-}=$id. The decomposition (2.41) is also easily checked.

Theorem 2.5. Let $\epsilon, \delta$ be positive numbers, and $\alpha, \beta, \rho, \sigma$ be functions in one variable, and $F^{+}, G^{-}, V^{+}, V^{-}$be the loops given by (2.38), (2.42), 2.43). Define a, b, c, r, s, $t$ by (2.39), (2.40), and $\hat{F}$ by $\hat{F}_{n}^{m}=F_{n}^{+} V_{n, m}^{-}=G_{m}^{-} V_{n, m}^{+}$. We assume that sequences $\alpha$, $\rho$ and $1-b$ save no zeros. Then there exists a diagonal matrix $D_{n}^{m}$ such that $\left(D_{0}^{0}\right)^{-1} \hat{F}_{n}^{m} D_{n}^{m}$ is the extended frame of some discrete indefinite improper affine sphere $f$, whose data solving the discrete Liouville equation 2.36 are given as

$$
\omega_{n}^{m}=\left(1-b_{n} s_{m}\right) \alpha_{n+1} \rho_{m+1}, \quad A_{n}=\alpha_{n+1} \alpha_{n} \beta_{n}, \quad B_{m}=\rho_{m+1} \rho_{m} \sigma_{m} .
$$

Moreover, the associated family of $f$ is given by the representation formula

$$
f_{n}^{m}=\left[\begin{array}{c}
\lambda a_{n}+\lambda^{-2}\left(r_{m} s_{m}-t_{m}\right) \\
\lambda^{2}\left(a_{n} b_{n}-c_{n}\right)+\lambda^{-1} r_{m} \\
a_{n} r_{m}-\left(a_{n} b_{n}-c_{n}\right)\left(r_{m} s_{m}-t_{m}\right)+\lambda^{3} \epsilon \sum_{k}^{n} \alpha_{k} c_{k-1}+\lambda^{-3} \delta \sum_{k}^{m} \rho_{k} t_{k-1}
\end{array}\right],
$$


where $\lambda \in \mathbb{R}^{\times}$. All discrete indefinite improper affine spheres are locally constructed in this way.

Proof. First a straightforward computation shows that Maurer-Cartan form of $\hat{F}$ is computed as

$$
\begin{aligned}
\left(\hat{F}_{n}^{m}\right)^{-1} \hat{F}_{n+1}^{m} & =\left(V_{n, m}^{-}\right)^{-1} \xi_{n}^{+} V_{n+1, m}^{-} \\
& =\left[\begin{array}{ccr}
1 & 0 & 0 \\
\frac{\beta_{n+1}}{\left(1-b_{n} s_{m}\right)\left(1-b_{n+1} s_{m}\right)} \epsilon \lambda & 1 & 0 \\
\frac{\alpha_{n+1} \beta_{n+1}}{1-b_{n+1} s_{m}}(\epsilon \lambda)^{2} & \alpha_{n+1}\left(1-b_{n} s_{m}\right) \epsilon \lambda & 1
\end{array}\right], \\
\left(\hat{F}_{n}^{m}\right)^{-1} \hat{F}_{n}^{m+1} & =\left(V_{n, m}^{+}\right)^{-1} \xi_{m}^{-} V_{n, m+1}^{+} \\
& =\left[\begin{array}{ccc}
\frac{1-b_{n} s_{m}}{1-b_{n} s_{m+1}} & \sigma_{m+1} \delta \lambda^{-1} & 0 \\
0 & \frac{1-b_{n} s_{m+1}}{1-b_{n} s_{m}} & 0 \\
\frac{\rho_{m+1}\left(1-b_{n} s_{m}\right)}{1-b_{n} s_{m+1}} \delta \lambda^{-1} & \rho_{m+1} \sigma_{m+1}\left(\delta \lambda^{-1}\right)^{2} & 1
\end{array}\right] .
\end{aligned}
$$

Next we take a diagonal gauge $D_{n}^{m}=\operatorname{diag}\left(d_{n}^{m}, 1 / d_{n}^{m}, 1\right)$ so that the Maurer-Cartan form of $F_{n}^{m}=\hat{F}_{n}^{m} D_{n}^{m}$ is

$$
\begin{aligned}
\left(F_{n}^{m}\right)^{-1} F_{n+1}^{m} & =\left[\begin{array}{ccc}
\frac{d_{n+1}^{m}}{d_{n}^{m}} & 0 & 0 \\
\frac{\beta_{n+1} d_{n+1}^{m} d_{n}^{m}}{\left(1-b_{n} s_{m}\right)\left(1-b_{n+1} s_{m}\right)} \epsilon \lambda & \frac{d_{n}^{m}}{d_{n+1}^{m}} & 0 \\
\frac{\alpha_{n+1} \beta_{n+1} d_{n+1}^{m}(\epsilon \lambda)^{2}}{1-b_{n+1} s_{m}}(\epsilon \lambda & \frac{\alpha_{n+1}\left(1-b_{n} s_{m}\right)}{d_{n+1}^{m}} \epsilon \lambda & 1
\end{array}\right], \\
\left(F_{n}^{m}\right)^{-1} F_{n}^{m+1} & =\left[\begin{array}{ccc}
\frac{d_{n}^{m+1}}{d_{n}^{m}} \frac{1-b_{n} s_{m}}{1-b_{n} s_{m+1}} & \frac{\sigma_{m+1}}{d_{n}^{m+1} d_{n}^{m}} \delta \lambda^{-1} & 0 \\
0 & \frac{d_{m}^{m}}{d_{n}^{m+1} \frac{1-b_{n} s_{m+1}}{1-b_{n} s_{m}}} & 0 \\
\frac{\rho_{m+1}\left(1-b_{n} s_{m}\right) d_{n}^{m+1}}{1-b_{n} s_{m+1}} \delta \lambda^{-1} & \frac{\rho_{m+1} \sigma_{m+1}}{d_{n}^{m+1}}\left(\delta \lambda^{-1}\right)^{2} & 1
\end{array}\right] .
\end{aligned}
$$

This should be compared with $2.13-2.14$ with $H=0$, thus we have 2.44 and

$$
d_{n}^{m}=\left(1-b_{n} s_{m}\right) \alpha_{n+1}
$$

To obtain the formula 2.45), we consider another diagonal gauge as introduced in 2.11). Namely, setting $\tilde{F}_{n}^{m}=F_{n}^{m} \tilde{D}_{n}^{m}$ where $\tilde{D}_{n}^{m}=\operatorname{diag}\left(\lambda, \lambda^{-1} \omega_{n}^{m}, 1\right)$, we have

$$
\begin{aligned}
& \left(\tilde{F}_{n}^{m}\right)^{-1} \tilde{F}_{n+1}^{m}=\left[\begin{array}{ccc}
\frac{\alpha_{n+2}}{\alpha_{n+1}} \frac{1-b_{n+1} s_{m}}{1-b_{n} s_{m}} & 0 & 0 \\
\frac{\alpha_{n+2} \beta_{n+1} \epsilon}{\rho_{m+1}\left(1-b_{n} s_{m}\right)} \lambda^{3} & 1 & 0 \\
\alpha_{n+2} \alpha_{n+1} \beta_{n+1} \epsilon^{2} \lambda^{3} & \alpha_{n+1} \rho_{m+1}\left(1-b_{n} s_{m}\right) \epsilon & 1
\end{array}\right]=\tilde{U}_{n}^{m} \\
& \left(\tilde{F}_{n}^{m}\right)^{-1} \tilde{F}_{n}^{m+1}=\left[\begin{array}{ccc}
1 & \frac{\sigma_{m+1} \rho_{m+2}}{\alpha_{n+1}\left(1-b_{n} s_{m}\right)} \delta \lambda^{-3} & 0 \\
0 & \frac{\rho_{m+2}\left(1-b_{n} s_{m+1}\right)}{\rho_{m+1}\left(1-b_{n} s_{m}\right)} & 0 \\
\alpha_{n+1} \rho_{m+1}\left(1-b_{n} s_{m}\right) \delta & \rho_{m+2} \rho_{m+1} \sigma_{m+1} \delta^{2} \lambda^{-3} & 1
\end{array}\right]=\tilde{V}_{n}^{m} .
\end{aligned}
$$

Of course this pair of matrices $\left(\tilde{U}_{n}^{m}, \tilde{V}_{n}^{m}\right)$ accords with 2.9 and 2.10 . The frame $\tilde{F}$ can be computed explicitly as

$$
\tilde{F}_{n}^{m}=G_{m}^{-} V_{n, m}^{+} D_{n}^{m} \tilde{D}_{n}^{m}=\left[v_{n}^{m}, w_{n}^{m}, \xi_{0}\right]
$$


where

$$
\begin{aligned}
v_{n}^{m}=\alpha_{n+1} & {\left[\begin{array}{c}
\lambda \\
b_{n} \lambda^{2} \\
r_{m}-b_{n}\left(r_{m} s_{m}-t_{m}\right)+c_{n} \lambda^{3}
\end{array}\right], } \\
w_{n}^{m}=\rho_{m+1} & {\left[\begin{array}{c}
s_{m} \lambda^{-2} \\
\lambda^{-1} \\
a_{n}-s_{m}\left(a_{n} b_{n}-c_{n}\right)+t_{m} \lambda^{-3}
\end{array}\right] . }
\end{aligned}
$$

By definition of the moving frame, we have

$$
v_{n}^{m}=\frac{f_{n+1}^{m}-f_{n}^{m}}{\epsilon}, \quad w_{n}^{m}=\frac{f_{n}^{m+1}-f_{n}^{m}}{\delta} .
$$

Hence, noticing (2.37), $a_{0} b_{0}=0$ and the relations

$$
\begin{aligned}
a_{n} b_{n}-c_{n} & =a_{n} b_{n}-\epsilon \sum_{k}^{n} a_{k} \beta_{k} \\
& =a_{n} b_{n}-\sum_{k}^{n} a_{k}\left(b_{k}-b_{k-1}\right) \\
& =\sum_{k}^{n}\left(a_{k}-a_{k-1}\right) b_{k-1} \\
& =\epsilon \sum_{k}^{n} \alpha_{k} b_{k-1}
\end{aligned}
$$

and $r_{m} s_{m}-t_{m}=\delta \sum_{k}^{m} \rho_{k} s_{k-1}$, it follows for all integers $n$ and $m$ that

$$
\begin{aligned}
& f_{n}^{m}-f_{0}^{0} \\
& =\sum_{k}^{n}\left(f_{k}^{0}-f_{k-1}^{0}\right)+\sum_{l}^{m}\left(f_{n}^{l}-f_{n}^{l-1}\right) \\
& =\epsilon \sum_{k}^{n} v_{k-1}^{0}+\delta \sum_{l}^{m} w_{n}^{l-1} \\
& =\epsilon \sum_{k}^{n} \alpha_{k}\left[\begin{array}{c}
\lambda \\
b_{k-1} \lambda^{2} \\
c_{k-1} \lambda^{3}
\end{array}\right]+\delta \sum_{l}^{m} \rho_{l}\left[\begin{array}{c}
s_{l-1} \lambda^{-2} \\
\lambda^{-1} \\
a_{n}-s_{l-1}\left(a_{n} b_{n}-c_{n}\right)+t_{l-1} \lambda^{-3}
\end{array}\right] \\
& \lambda a_{n}+\lambda^{-2}\left(r_{m} s_{m}-t_{m}\right) \\
& \lambda^{2}\left(a_{n} b_{n}-c_{n}\right)+\lambda^{-1} r_{m} \\
& =\left[\begin{array}{c}
\left.r_{m} s_{m}-t_{m}\right)+\lambda^{3} \epsilon \sum_{k}^{n} \alpha_{k} c_{k-1}+\lambda^{-3} \delta \sum_{l}^{m} \rho_{l} t_{l-1}
\end{array}\right] .
\end{aligned}
$$

Thus we have the representation formula 2.45) up to equiaffine transformations.

Remark 2.6. For given functions $A_{n}, B_{m}$ and positive constants $\epsilon, \delta$, it is known that a general solution to the discrete Liouville equation (2.36) can be expressed as

$$
\omega_{n}^{m}=\frac{\epsilon\left(p_{0}+\sum_{k}^{n} A_{k} \phi_{k} \phi_{k-1}\right)+\delta\left(q_{0}+\sum_{l}^{m} B_{l} \psi_{l} \psi_{l-1}\right)}{\phi_{n} \psi_{m}},
$$

where $\phi$ and $\psi$ are arbitrary functions with no zeros in one variable, and $p_{0}$ and $q_{0}$ are arbitrary constants. See, for example, [24]. On the other hand, our formula (2.44) tells that a general solution also has an expression

$$
\omega_{n}^{m}=\alpha_{n+1} \rho_{m+1}\left(1-\epsilon \delta \sum_{k}^{n} \frac{A_{k}}{\alpha_{k+1} \alpha_{k}} \sum_{l}^{m} \frac{B_{l}}{\rho_{l+1} \rho_{l}}\right),
$$

where $\alpha$ and $\rho$ are arbitrary functions with no zeros in one variable. As the most simple solutions, by setting $\phi=\psi=\alpha=\rho=1$ and $p_{0}=q_{0}=0$, these formulas give an additive one $\omega_{n}^{m}=\epsilon \sum_{k}^{n} A_{k}+\delta \sum_{l}^{m} B_{l}$ and a multiplicative one $\omega_{n}^{m}=1-\epsilon \delta \sum_{k}^{n} A_{k} \sum_{l}^{m} B_{l}$, respectively.

We conclude this paper with one of our main results, which offers a representation formula using two discrete plane curves for a discrete indefinite improper affine sphere. 
Corollary 2.7 (Representation formula). Fix positive numbers $\epsilon$ and $\delta$. For maps $a, p: \epsilon \mathbb{Z} \rightarrow$ $\mathbb{R}$ and $q, r: \delta \mathbb{Z} \rightarrow \mathbb{R}$, set

Then the map

$$
\gamma_{n}^{1}=\left[\begin{array}{l}
a_{n} \\
p_{n}
\end{array}\right], \quad \gamma_{m}^{2}=\left[\begin{array}{l}
q_{m} \\
r_{m}
\end{array}\right]
$$

$$
f_{n}^{m}=\left[\begin{array}{c}
\gamma_{n}^{1}+\gamma_{m}^{2} \\
z_{n}^{m}
\end{array}\right]
$$

where

$$
z_{n}^{m}=\operatorname{det}\left[\gamma_{n}^{1}, \gamma_{m}^{2}\right]+\sum_{k}^{n} \operatorname{det}\left[\gamma_{k-1}^{1}, \gamma_{k}^{1}\right]-\sum_{k}^{m} \operatorname{det}\left[\gamma_{k-1}^{2}, \gamma_{k}^{2}\right]
$$

is a discrete indefinite improper affine sphere with the affine normal ${ }^{\mathrm{t}}[0,0,1]$. Its data solving the discrete Liouville equation 2.36) is

$$
\begin{aligned}
& \omega_{n}^{m}=\operatorname{det}\left[\frac{\gamma_{n+1}^{1}-\gamma_{n}^{1}}{\epsilon}, \frac{\gamma_{m+1}^{2}-\gamma_{m}^{2}}{\delta}\right], \\
& A_{n}=\operatorname{det}\left[\frac{\gamma_{n+1}^{1}-\gamma_{n}^{1}}{\epsilon},-\frac{\gamma_{n}^{1}-\gamma_{n-1}^{1}}{\epsilon^{2}}\right], \\
& B_{m}=\operatorname{det}\left[-\frac{\gamma_{m}^{2}-\gamma_{m-1}^{2}}{\delta^{2}}, \frac{\gamma_{m+1}^{2}-\gamma_{m}^{2}}{\delta}\right] .
\end{aligned}
$$

Moreover the associated family of $f$ is given by the transformation

$$
\gamma^{1} \mapsto\left[\begin{array}{cc}
\lambda & 0 \\
0 & \lambda^{2}
\end{array}\right] \gamma^{1}, \quad \gamma^{2} \mapsto\left[\begin{array}{cc}
\lambda^{-2} & 0 \\
0 & \lambda^{-1}
\end{array}\right] \gamma^{2}
$$

where $\lambda \in \mathbb{R}^{\times}$. Conversely all discrete indefinite improper affine spheres can be constructed in this way.

Proof. First, introducing functions $p_{n}=a_{n} b_{n}-c_{n}$ and $q_{m}=r_{m} s_{m}-t_{m}$, we rephrase 2.45) as

$$
f_{n}^{m}=\left[\begin{array}{c}
\lambda a_{n}+\lambda^{-2} q_{m} \\
\lambda^{2} p_{n}+\lambda^{-1} r_{m} \\
a_{n} r_{m}-p_{n} q_{m}+\lambda^{3} \sum_{k}^{n}\left(a_{k-1} p_{k}-a_{k} p_{k-1}\right)-\lambda^{-3} \sum_{k}^{m}\left(r_{k} q_{k-1}-r_{k-1} q_{k}\right)
\end{array}\right],
$$

where we use the identities

$$
\begin{aligned}
\epsilon \alpha_{k} c_{k-1} & =\left(a_{k}-a_{k-1}\right) c_{k-1} \\
& =a_{k} c_{k-1}+a_{k-1}\left(\epsilon a_{k} \beta_{k}-c_{k}\right) \\
& =a_{k} c_{k-1}+a_{k} a_{k-1}\left(b_{k}-b_{k-1}\right)-a_{k-1} c_{k} \\
& =a_{k-1} p_{k}-a_{k} p_{k-1},
\end{aligned}
$$

and $\delta \rho_{k} t_{k-1}=r_{k-1} q_{k}-r_{k} q_{k-1}$. Note that $a_{0}=p_{0}=q_{0}=r_{0}=0$. We then consider an equiaffine transformation of $f_{n}^{m}$ as

$$
\tilde{f}_{n}^{m}=\left[\begin{array}{ccc}
1 & 0 & 0 \\
0 & 1 & 0 \\
\lambda^{-1} \tilde{r}_{0}-\lambda^{2} \tilde{p}_{0} & \lambda \tilde{a}_{0}-\lambda^{-2} \tilde{q}_{0} & 1
\end{array}\right] f_{n}^{m}+\left[\begin{array}{c}
\lambda \tilde{a}_{0}+\lambda^{-2} \tilde{q}_{0} \\
\lambda^{2} \tilde{p}_{0}+\lambda^{-1} \tilde{r}_{0} \\
\tilde{a}_{0} \tilde{r}_{0}-\tilde{p}_{0} \tilde{q}_{0}
\end{array}\right],
$$

where $\tilde{a}_{0}, \tilde{r}_{0}, \tilde{p}_{0}$ and $\tilde{q}_{0}$ are some constants. A straightforward computation shows that

$$
\tilde{f}_{n}^{m}=\left[\begin{array}{c}
\lambda \tilde{a}_{n}+\lambda^{-2} \tilde{q}_{m} \\
\lambda^{2} \tilde{p}_{n}+\lambda^{-1} \tilde{r}_{m} \\
\tilde{a}_{n} \tilde{r}_{m}-\tilde{p}_{n} \tilde{q}_{m}+\lambda^{3} \sum_{k}^{n}\left(\tilde{a}_{k-1} \tilde{p}_{k}-\tilde{a}_{k} \tilde{p}_{k-1}\right)-\lambda^{-3} \sum_{k}^{m}\left(\tilde{r}_{k} \tilde{q}_{k-1}-\tilde{r}_{k-1} \tilde{q}_{k}\right)
\end{array}\right],
$$


where $\tilde{a}_{n}=a_{n}+\tilde{a}_{0}, \tilde{p}_{n}=p_{n}+\tilde{p}_{0}, \tilde{q}_{n}=q_{n}+\tilde{q}_{0}$ and $\tilde{r}=r+\tilde{r}_{0}$. Thus we obtain 2.48) on writing

$$
\gamma_{n}^{1}=\left[\begin{array}{l}
\tilde{a}_{n} \\
\tilde{p}_{n}
\end{array}\right], \quad \gamma_{m}^{2}=\left[\begin{array}{l}
\tilde{q}_{m} \\
\tilde{r}_{m}
\end{array}\right] .
$$

Since $\gamma_{n}^{1}$ and $\gamma_{m}^{2}$ are arbitrary, 2.48 gives the all improper indefinite affine spheres.

Remark 2.8. We recall that the height function $z$ defined by (1.31) satisfies $\partial_{u} \partial_{v} z(u, v)=$ $\operatorname{det}\left[\gamma_{1}^{\prime}(u), \gamma_{2}^{\prime}(v)\right]$. As a discrete analogue of this, the sequence $z$ defined by (2.49) satisfies a difference equation

$$
z_{n+1}^{m+1}-z_{n+1}^{m}-z_{n}^{m+1}+z_{n}^{m}=\operatorname{det}\left[\gamma_{n+1}^{1}-\gamma_{n}^{1}, \gamma_{m+1}^{2}-\gamma_{m}^{2}\right] .
$$

In particular, if $\epsilon=\delta$ and $\gamma_{i}^{1}=\gamma_{i}^{2}$ for all $i \in \mathbb{Z}$, then $z$ satisfies $z_{i}^{i}=z_{i}^{i \pm 1}=0$ for all $i \in \mathbb{Z}$, and then every $z_{i}^{i \pm 2}$ can be fast computed by using the recurrent relation (2.51). Iterating this, we can obtain all the values $z_{n}^{m}$ numerically. Refer to Example 4 for specific examples, where we explicitly calculate $z$ as a function in $(n, m)$.

We fix positive numbers $\epsilon$ and $\delta$ arbitrarily to illustrate examples of discrete indefinite improper affine spheres by taking several discrete curves.

Example 3. Let $P_{n}$ and $R_{m}$ be arbitrary sequences which possibly depend on $\epsilon$ and $\delta$ respectively. We denote by $\Delta P$ and $\Delta R$ the forward differences of them, that is,

$$
\Delta P_{n}=\frac{P_{n+1}-P_{n}}{\epsilon}, \quad \Delta R_{m}=\frac{R_{m+1}-R_{m}}{\delta} .
$$

We substitute discrete curves

$$
\gamma_{n}^{1}=\left[\begin{array}{c}
\epsilon n \\
\Delta P_{n}
\end{array}\right], \quad \gamma_{m}^{2}=\left[\begin{array}{c}
\Delta R_{m} \\
\delta m
\end{array}\right]
$$

into the representation formula (2.48), and have a discrete indefinite improper affine sphere

$$
f_{n}^{m}=\left[\begin{array}{c}
\epsilon n+\Delta R_{m} \\
\delta m+\Delta P_{n} \\
\left(\epsilon n+\Delta R_{m}\right)\left(\delta m+\Delta P_{n}\right)-2\left(\Delta P_{n}\right)\left(\Delta R_{m}\right)-P_{n+1}-P_{n}-R_{m+1}-R_{m}
\end{array}\right] .
$$

Its data is

$$
\begin{gathered}
\omega_{n}^{m}=1-\epsilon^{-2} \delta^{-2}\left(P_{n+2}-2 P_{n+1}+P_{n}\right)\left(R_{m+2}-2 R_{m+1}+R_{m}\right), \\
A_{n}=\epsilon^{-3}\left(P_{n+2}-3 P_{n+1}+3 P_{n}-P_{n-1}\right), \\
B_{m}=\delta^{-3}\left(R_{m+2}-3 R_{m+1}+3 R_{m}-R_{m-1}\right) .
\end{gathered}
$$

Example 4. We illustrate discrete counterparts to those surfaces given in Example 2, Fix positive numbers $q_{1}, q_{2}$ arbitrarily and introduce positive numbers

$$
\theta_{1}=\frac{2}{\epsilon} \arctan \left(\frac{\epsilon}{2} q_{1}\right), \quad \theta_{2}=\frac{2}{\delta} \arctan \left(\frac{\delta}{2} q_{2}\right) .
$$

(1) First example is given by the discrete curves

$$
\gamma_{n}^{1}=\left[\begin{array}{c}
\cos \left(\theta_{1} \epsilon n\right) \\
\sin \left(\theta_{1} \epsilon n\right)
\end{array}\right], \quad \gamma_{m}^{2}=\left[\begin{array}{c}
\cos \left(\theta_{2} \delta m\right) \\
\sin \left(\theta_{2} \delta m\right)
\end{array}\right]
$$

Substituting these into the representation formula, we have

$$
f_{n}^{m}=\left[\begin{array}{c}
\cos \left(\theta_{1} \epsilon n\right)+\cos \left(\theta_{2} \delta m\right) \\
\sin \left(\theta_{1} \epsilon n\right)+\sin \left(\theta_{2} \delta m\right) \\
-\sin \left(\theta_{1} \epsilon n-\theta_{2} \delta m\right)+n \sin \left(\theta_{1} \epsilon\right)-m \sin \left(\theta_{2} \delta\right)
\end{array}\right] .
$$


The data solving the discrete Liouville equation 2.36 are given by constants

$$
\begin{gathered}
A_{n}=\frac{8}{\epsilon^{3}} \sin ^{3}\left(\frac{\epsilon}{2} \theta_{1}\right) \cos \left(\frac{\epsilon}{2} \theta_{1}\right)=\frac{16 q_{1}^{3}}{\left(4+\epsilon^{2} q_{1}^{2}\right)^{2}}, \\
B_{m}=-\frac{8}{\delta^{3}} \sin ^{3}\left(\frac{\delta}{2} \theta_{2}\right) \cos \left(\frac{\delta}{2} \theta_{2}\right)=-\frac{16 q_{2}^{3}}{\left(4+\delta^{2} q_{2}^{2}\right)^{2}},
\end{gathered}
$$

and a sequence

$$
\omega_{n}^{m}=\frac{4 q_{1} q_{2}}{\sqrt{4+\epsilon^{2} q_{1}^{2}} \sqrt{4+\delta^{2} q_{2}^{2}}} \sin \left(\frac{\epsilon}{2} \theta_{1}(2 n+1)-\frac{\delta}{2} \theta_{2}(2 m+1)\right) .
$$

Therefore $f$ is singular if $n, m$ satisfy

$$
\epsilon \theta_{1}(2 n+1) \equiv \delta \theta_{2}(2 m+1) \quad(\bmod 2 \pi) .
$$
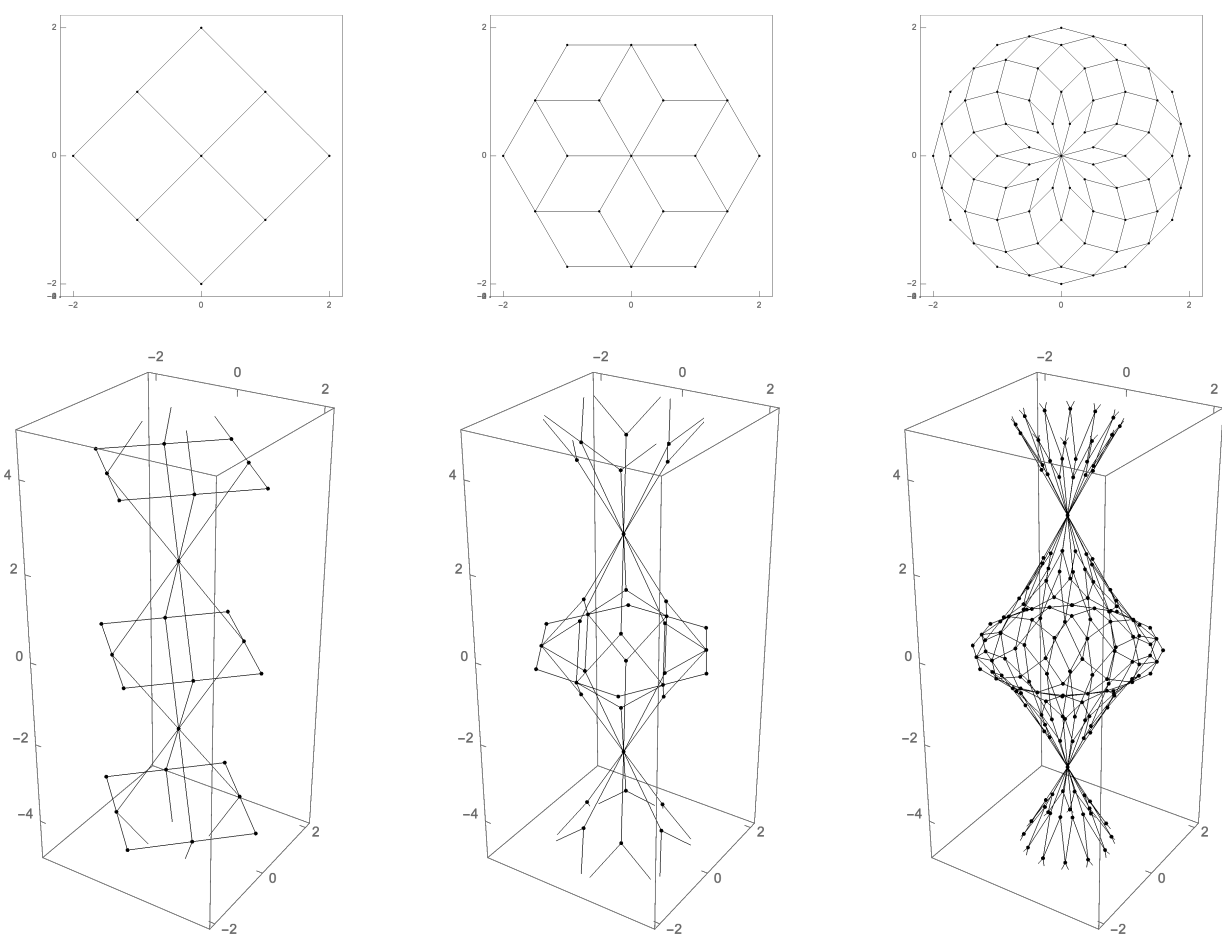

(d) $q=2 \tan (\pi / 4)$

(e) $q=2 \tan (\pi / 6)$

(f) $q=2 \tan (\pi / 12)$

Figure 5. Discrete indefinite improper affine spheres $f_{n}^{m}$ with $\epsilon=\delta=1$ and $q=q_{1}=q_{2}$, which exhibit cone points. The figures in upper line show views from the top.

(2) Second example is given by

$$
\gamma_{n}^{1}=\left[\begin{array}{c}
\left|\cos \left(\theta_{1} \epsilon n\right)\right| \cos \left(\theta_{1} \epsilon n\right) \\
\left|\sin \left(\theta_{1} \epsilon n\right)\right| \sin \left(\theta_{1} \epsilon n\right)
\end{array}\right], \quad \gamma_{m}^{2}=\left[\begin{array}{c}
\left|\cos \left(\theta_{2} \delta m\right)\right| \cos \left(\theta_{2} \delta m\right) \\
\left|\sin \left(\theta_{2} \delta m\right)\right| \sin \left(\theta_{2} \delta m\right)
\end{array}\right] .
$$

We assume that both the images of $\gamma^{1}$ and $\gamma^{2}$ contain four points

$$
\pm\left[\begin{array}{l}
1 \\
0
\end{array}\right], \quad \pm\left[\begin{array}{l}
0 \\
1
\end{array}\right]
$$


which we can always achieve by choosing $q_{1}, q_{2}$ appropriately. By virtue of this assumption, we are able to assume that $0<\theta_{1} \epsilon \leq \pi / 2$ and $\cos \left(\theta_{1} \epsilon(n-1)\right) \cos \left(\theta_{1} \epsilon n\right) \geq$ 0 and $\sin \left(\theta_{1} \epsilon(n-1)\right) \sin \left(\theta_{1} \epsilon n\right) \geq 0$. Therefore the differences of $\gamma^{1}$ can be written into simple forms as

$$
\gamma_{n}^{1}-\gamma_{n-1}^{1}=2 \sin \left(\theta_{1} \epsilon\right)\left[\begin{array}{c}
-\left|\cos \frac{\theta_{1} \epsilon(2 n-1)}{2}\right| \sin \frac{\theta_{1} \epsilon(2 n-1)}{2} \\
\left|\sin \frac{\theta_{1} \epsilon(2 n-1)}{2}\right| \cos \frac{\theta_{1} \epsilon(2 n-1)}{2}
\end{array}\right] .
$$

Hence we have that

$$
\operatorname{det}\left[\gamma_{n-1}^{1}, \gamma_{n}^{1}\right]=\operatorname{det}\left[\gamma_{n}^{1}, \gamma_{n}^{1}-\gamma_{n-1}^{1}\right]=\sin \left(\theta_{1} \epsilon\right)\left|\sin \left(\theta_{1} \epsilon(2 n-1)\right)\right| .
$$

Further, on choosing the parameter $q_{1}$ as

$$
q_{1}=\frac{2}{\epsilon} \tan \frac{\pi}{4 N_{1}}
$$

with a positive integer $N_{1}$, which is equivalent to setting $\theta_{1} \epsilon=\pi /\left(2 N_{1}\right)$, it holds for all $n \in \mathbb{Z}$ that

$$
\begin{aligned}
\sum_{k}^{n} \operatorname{det}\left(\gamma_{k-1}^{1}, \gamma_{k}^{1}\right) & =\sum_{k}^{n} \sin \frac{\pi}{2 N_{1}}\left|\sin \frac{(2 k-1) \pi}{2 N_{1}}\right| \\
& =\left\lfloor\frac{n}{N_{1}}\right\rfloor+\frac{1}{2}\left(1-(-1)^{\left\lfloor n / N_{1}\right\rfloor} \cos \frac{n \pi}{N_{1}}\right) .
\end{aligned}
$$

Here $\lfloor u\rfloor$ is the floor of $u$, that is, the greatest integer less than or equal to $u$. We apply the same discussion as above to $\gamma^{2}$, and set $q_{2}=(2 / \delta) \tan \left(\pi /\left(4 N_{2}\right)\right)$ to have

$$
\begin{gathered}
f_{n}^{m}=\left[\begin{array}{r}
\left.\left|\cos \frac{n \pi}{2 N_{1}}\right| \cos \frac{n \pi}{2 N_{1}}+\mid \begin{array}{c}
\cos \frac{m \pi}{2 N_{2}} \mid \cos \frac{m \pi}{2 N_{2}} \\
\sin \frac{n \pi}{2 N_{1}}\left|\sin \frac{n \pi}{2 N_{1}}+\right|
\end{array}\right] \sin \frac{m \pi}{2 N_{2}} \mid \sin \frac{m \pi}{2 N_{2}} \\
z_{n}^{m}
\end{array}\right], \\
z_{n}^{m}=\left\lfloor\cos \frac{n \pi}{2 N_{1}} \sin \frac{m \pi}{2 N_{2}}\left|\cos \frac{n \pi}{2 N_{1}} \sin \frac{m \pi}{2 N_{2}}-\right| \sin \frac{n \pi}{2 N_{1}} \cos \frac{m \pi}{2 N_{2}} \mid \sin \frac{n \pi}{2 N_{1}} \cos \frac{m \pi}{2 N_{2}}\right. \\
+\left\lfloor\frac{n}{N_{1}}\right\rfloor-\left\lfloor\frac{m}{N_{2}}\right\rfloor-\frac{1}{2}\left((-1)^{\left\lfloor n / N_{1}\right\rfloor} \cos \frac{n \pi}{N_{1}}-(-1)^{\left\lfloor m / N_{2}\right\rfloor} \cos \frac{m \pi}{N_{2}}\right) .
\end{gathered}
$$

Its data is

$$
\begin{gathered}
A_{n}= \begin{cases}\frac{2}{\epsilon^{3}} \sin ^{4} \frac{\pi}{2 N_{1}} & n \in N_{1} \mathbb{Z} \\
0 & n \notin N_{1} \mathbb{Z},\end{cases} \\
B_{m}= \begin{cases}-\frac{2}{\delta^{3}} \sin ^{4} \frac{\pi}{2 N_{2}} & m \in N_{2} \mathbb{Z} \\
0 & m \notin N_{2} \mathbb{Z},\end{cases} \\
\omega_{n}^{m}=\frac{4}{\epsilon \delta} \sin \frac{\pi}{2 N_{1}} \sin \frac{\pi}{2 N_{2}}\left(\left|\cos \frac{(2 n+1) \pi}{4 N_{1}} \sin \frac{(2 m+1) \pi}{4 N_{2}}\right| \sin \frac{(2 n+1) \pi}{4 N_{1}} \cos \frac{(2 m+1) \pi}{4 N_{2}}\right. \\
\left.-\cos \frac{(2 n+1) \pi}{4 N_{1}} \sin \frac{(2 m+1) \pi}{4 N_{2}}\left|\sin \frac{(2 n+1) \pi}{4 N_{1}} \cos \frac{(2 m+1) \pi}{4 N_{2}}\right|\right) \\
= \begin{cases}0 & (n, m) \in S \\
\frac{2}{\epsilon \delta} \sin \frac{\pi}{2 N_{1}} \sin \frac{\pi}{2 N_{2}} \sin \frac{(2 n+1) \pi}{2 N_{1}} \sin \frac{(2 m+1) \pi}{2 N_{2}} & (n, m) \notin S .\end{cases}
\end{gathered}
$$

The singular set $S$ consists of integer points in a checkerboard, that is

$$
S=\left\{(n, m) \in \mathbb{Z}^{2} \mid\left\lfloor n / N_{1}\right\rfloor \equiv\left\lfloor m / N_{2}\right\rfloor(\bmod 2)\right\} .
$$



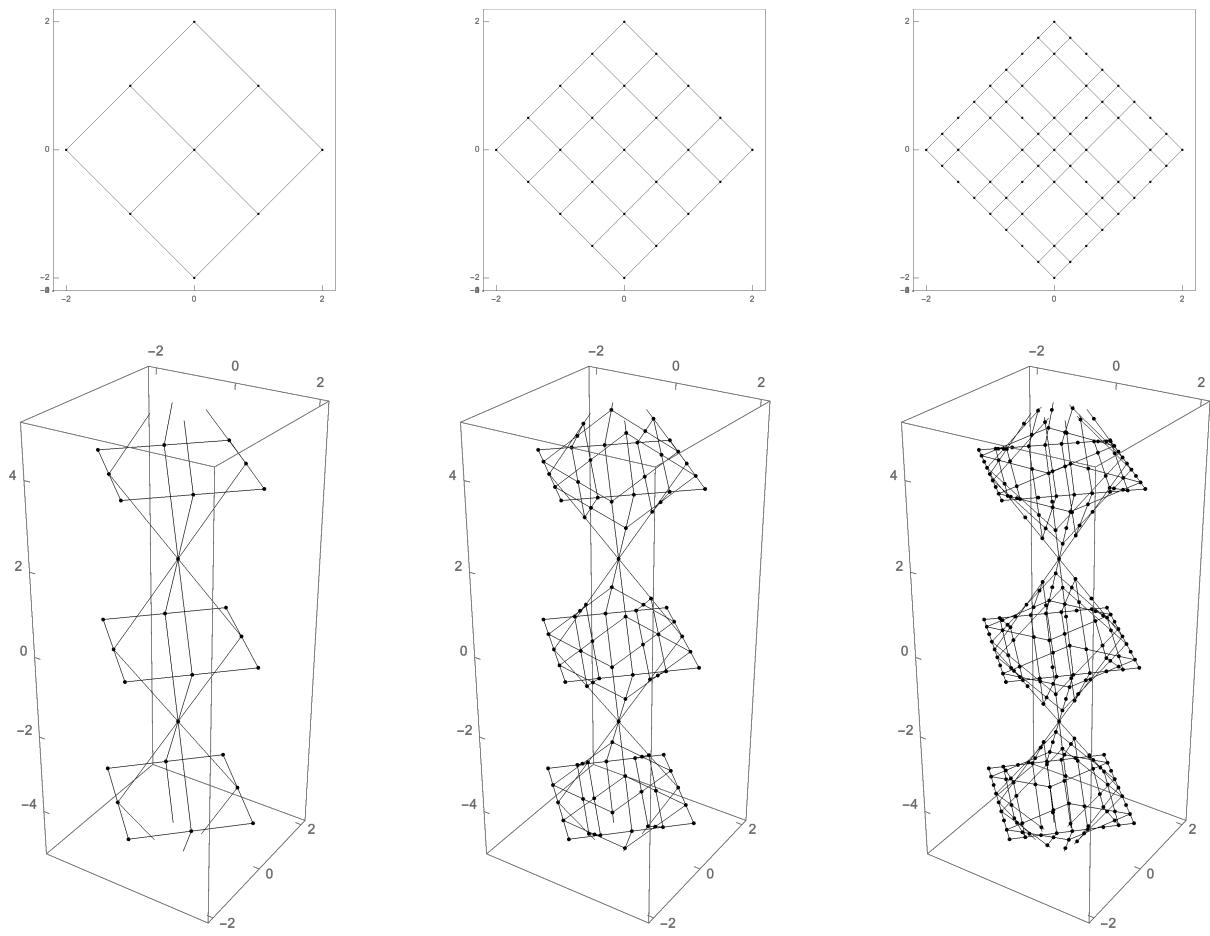

(d) $N=1$

(e) $N=2$

(f) $N=3$

Figure 6. Discrete indefinite improper affine spheres $f_{n}^{m}$ with $\epsilon=\delta=1$ and $N=N_{1}=N_{2}$. The figures in upper line show views from the top.

(3) Third example is given by

$$
\gamma_{n}^{1}=\cos \left(\theta_{1} \epsilon n\right)\left[\begin{array}{c}
1 / 2+\cos ^{2}\left(\theta_{1} \epsilon n\right) \\
2 \sin \left(\theta_{1} \epsilon n\right)
\end{array}\right], \quad \gamma_{m}^{2}=\cos \left(\theta_{2} \delta m\right)\left[\begin{array}{c}
1 / 2+\cos ^{2}\left(\theta_{2} \delta m\right) \\
2 \sin \left(\theta_{2} \delta m\right)
\end{array}\right] .
$$

We have

$$
\begin{aligned}
\operatorname{det}\left[\gamma_{n-1}^{1}, \gamma_{n}^{1}\right]= & 2\left(3+2 \sin \left(\theta_{1} \epsilon n\right) \sin \left(\theta_{1} \epsilon(n-1)\right)\right) \sin \left(\theta_{1} \epsilon / 2\right) \\
& \cdot \cos \left(\theta_{1} \epsilon n\right) \cos \left(\theta_{1} \epsilon(n-1 / 2)\right) \cos \left(\theta_{1} \epsilon(n-1)\right),
\end{aligned}
$$

and hence

$$
\begin{aligned}
\sum_{k}^{n} \operatorname{det}\left[\gamma_{k-1}^{1}, \gamma_{k}^{1}\right]= & c_{1}\left(\theta_{1} \epsilon\right) \sin \left(\theta_{1} \epsilon n\right)+c_{2}\left(\theta_{1} \epsilon\right) \sin \left(3 \theta_{1} \epsilon n\right) \\
& +c_{3}\left(\theta_{1} \epsilon\right) \sin \left(5 \theta_{1} \epsilon n\right)
\end{aligned}
$$

Here the coefficients are given as

$$
\begin{aligned}
& c_{1}(t)=\frac{3+6 \cos t+\cos 2 t}{4}, \\
& c_{2}(t)=\frac{5}{8(1+2 \cos t)}, \\
& c_{3}(t)=-\frac{1}{8(1+2 \cos t+2 \cos 2 t)} .
\end{aligned}
$$


This should be compared with (1.38). We have

$f_{n}^{m}=\left[\begin{array}{c}\left(1+(1 / 2) \cos \left(2 \theta_{1} \epsilon n\right)\right) \cos \left(\theta_{1} \epsilon n\right)+\left(1+(1 / 2) \cos \left(2 \theta_{2} \delta m\right)\right) \cos \left(\theta_{2} \delta m\right) \\ \sin \left(2 \theta_{1} \epsilon n\right)+\sin \left(2 \theta_{2} \delta m\right) \\ z_{n}^{m}\end{array}\right]$,

where

$$
\begin{aligned}
z_{n}^{m}= & -\cos \left(\theta_{1} \epsilon n\right) \cos \left(\theta_{2} \delta m\right)\left(\sin \left(\theta_{1} \epsilon n\right)-\sin \left(\theta_{2} \delta m\right)\right) \\
& \cdot\left(3+2 \sin \left(\theta_{1} \epsilon n\right) \sin \left(\theta_{2} \delta m\right)\right) \\
& +c_{1}\left(\theta_{1} \epsilon\right) \sin \left(\theta_{1} \epsilon n\right)-c_{1}\left(\theta_{2} \delta\right) \sin \left(\theta_{2} \delta m\right) \\
& +c_{2}\left(\theta_{1} \epsilon\right) \sin \left(3 \theta_{1} \epsilon n\right)-c_{2}\left(\theta_{2} \delta\right) \sin \left(3 \theta_{2} \delta m\right) \\
& +c_{3}\left(\theta_{1} \epsilon\right) \sin \left(5 \theta_{1} \epsilon n\right)-c_{3}\left(\theta_{2} \delta\right) \sin \left(5 \theta_{2} \delta m\right)
\end{aligned}
$$

Its data is given as

$$
\begin{aligned}
A_{n}= & 4 \epsilon^{-3} \sin ^{3}\left(\theta_{1} \epsilon / 2\right) \cos \left(\theta_{1} \epsilon / 2\right)\left(a_{1}\left(\theta_{1} \epsilon / 2\right)\right. \\
& +a_{2}\left(\theta_{1} \epsilon / 2\right) \cos \left(2 \theta_{1} \epsilon n\right) \\
& \left.+a_{3}\left(\theta_{1} \epsilon / 2\right) \cos \left(4 \theta_{1} \epsilon n\right)\right) \cos \left(\theta_{1} \epsilon n\right), \\
B_{m}= & -4 \delta^{-3} \sin ^{3}\left(\theta_{2} \delta / 2\right) \cos \left(\theta_{2} \delta / 2\right)\left(a_{1}\left(\theta_{2} \delta / 2\right)\right. \\
& +a_{2}\left(\theta_{2} \delta / 2\right) \cos \left(2 \theta_{2} \delta m\right) \\
& \left.+a_{3}\left(\theta_{2} \delta / 2\right) \cos \left(4 \theta_{2} \delta m\right)\right) \cos \left(\theta_{2} \delta m\right), \\
\omega_{n}^{m}= & -4 \epsilon^{-1} \delta^{-1} \sin \left(\theta_{1} \epsilon / 2\right) \sin \left(\theta_{2} \delta / 2\right)\left(4 D_{n, m}^{1}\right. \\
& +8 D_{n, m}^{2} \sin \left(\theta_{1} \epsilon(n+1 / 2)\right) \sin \left(\theta_{2} \delta(m+1 / 2)\right) \\
& \left.+3 D_{n, m}^{3} \cos \left(\theta_{1} \epsilon(2 n+1)\right) \cos \left(\theta_{2} \delta(2 m+1)\right)\right),
\end{aligned}
$$

where

$$
\begin{aligned}
& a_{1}(t)=7+9 \cos 2 t+2 \cos 4 t+\cos 6 t \\
& a_{2}(t)=-6-2 \cos 2 t \\
& a_{3}(t)=1+2 \cos 2 t
\end{aligned}
$$

and

$$
\begin{aligned}
& D_{n, m}^{1}=\frac{3+\cos \left(\theta_{1} \epsilon\right)}{4} \cos \frac{\theta_{2} \delta}{2} \sin \frac{\theta_{1} \epsilon(2 n+1)}{2}-\frac{3+\cos \left(\theta_{2} \delta\right)}{4} \cos \frac{\theta_{1} \epsilon}{2} \sin \frac{\theta_{2} \delta(2 m+1)}{2}, \\
& D_{n, m}^{2}=\frac{3+\cos \left(\theta_{2} \delta\right)}{4} \cos \frac{\theta_{1} \epsilon}{2} \sin \frac{\theta_{1} \epsilon(2 n+1)}{2}-\frac{3+\cos \left(\theta_{1} \epsilon\right)}{4} \cos \frac{\theta_{2} \delta}{2} \sin \frac{\theta_{2} \delta(2 m+1)}{2}, \\
& D_{n, m}^{3}=\frac{1+2 \cos \left(\theta_{1} \epsilon\right)}{3} \cos \frac{\theta_{2} \delta}{2} \sin \frac{\theta_{1} \epsilon(2 n+1)}{2}-\frac{1+2 \cos \left(\theta_{2} \delta\right)}{3} \cos \frac{\theta_{1} \epsilon}{2} \sin \frac{\theta_{2} \delta(2 m+1)}{2} .
\end{aligned}
$$

Especially if we choose the parameters $q_{1}, q_{2}$ so as to be

$$
\theta_{1} \epsilon=\theta_{2} \delta=\frac{\pi}{N}
$$

with a positive integer $N$, then $\omega$ is factorized as

$$
\begin{aligned}
& \omega_{n}^{m}=-\frac{4}{\epsilon \delta} \sin ^{2} \frac{\pi}{2 N} \cos \frac{\pi}{2 N}\left(\sin \frac{\pi(2 n+1)}{2 N}-\sin \frac{\pi(2 m+1)}{2 N}\right) W_{n}^{m}, \\
& W_{n}^{m}=\left(3+\cos \frac{\pi}{N}\right)\left(1+2 \sin \frac{\pi(2 n+1)}{2 N} \sin \frac{\pi(2 m+1)}{2 N}\right) \\
&+\left(1+2 \cos \frac{\pi}{N}\right) \cos \frac{\pi(2 n+1)}{N} \cos \frac{\pi(2 m+1)}{N} \\
& 36
\end{aligned}
$$


The singular set $S=S_{1} \cup S_{2}$ is given by

$$
\begin{gathered}
S_{1}=\left\{(n, m) \in \mathbb{Z}^{2} \mid m \equiv n(\bmod 2 N), m \equiv-n+N-1(\bmod 2 N)\right\}, \\
S_{2}=\left\{(n, m) \in \mathbb{Z}^{2} \mid W_{n}^{m}=0\right\} .
\end{gathered}
$$

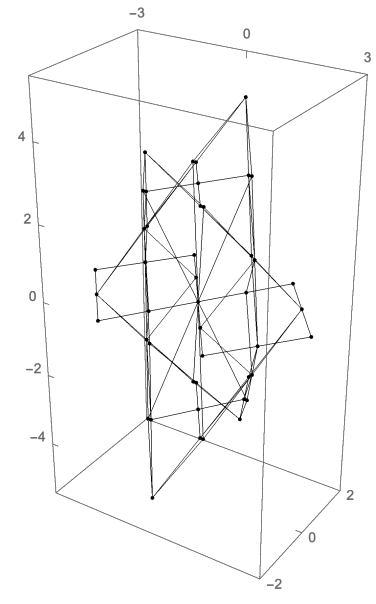

(a) $N=4$

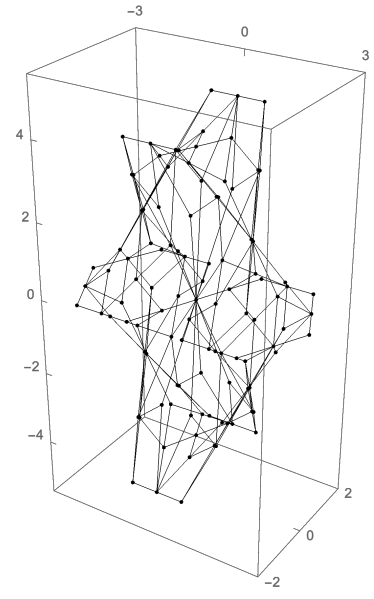

(b) $N=6$

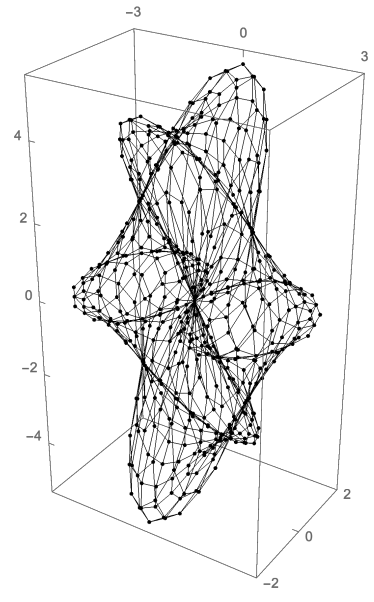

(c) $N=12$

FiguRE 7. Discrete indefinite improper affine spheres $f_{n}^{m}$ with $\epsilon=\delta=1$.

\section{REFERENCES}

[1] Wilhelm Blaschke, Vorlesungen über Differentialgeometrie und geometrische Grundlagen von Einsteins Relativitätstheorie. Band II. Affine Differentialgeometrie, Berlin, Verlag von Julius Springer, 1923.

[2] Alexander I. Bobenko and Wolfgang K. Schief, Affine spheres: discretization via duality relations, Experiment. Math. 8 (1999), no. 3, 261-280. MR 1724159

[3] _ Discrete indefinite affine spheres, Discrete integrable geometry and physics (Vienna, 1996), Oxford Lecture Ser. Math. Appl., vol. 16, Oxford Univ. Press, New York, 1999, pp. 113-138. MR 1676596

[4] Alexander I. Bobenko and Yuri B. Suris, Discrete differential geometry, Graduate Studies in Mathematics, vol. 98, American Mathematical Society, Providence, RI, 2008, Integrable structure. MR 2467378 (2010f:37125)

[5] Marcos Craizer, Moacyr Alvim, and Ralph Teixeira, Area distances of convex plane curves and improper affine spheres, SIAM J. Imaging Sci. 1 (2008), no. 3, 209-227. MR 2486030

[6] Marcos Craizer, Ralph C. Teixeira, and Moacyr A. H. B. da Silva, A geometric representation of improper indefinite affine spheres with singularities, J. Geom. 100 (2011), no. 1-2, 65-78. MR 2845277

[7] R. K. Dodd and R. K. Bullough, Polynomial conserved densities for the sine-Gordon equations, Proc. Roy. Soc. London Ser. A 352 (1977), no. 1671, 481-503. MR 0442516

[8] J. Dorfmeister and U. Eitner, Weierstraß-type representation of affine spheres, Abh. Math. Sem. Univ. Hamburg 71 (2001), 225-250. MR 1873045

[9] Roland Hildebrand, Canonical barriers on convex cones, Math. Oper. Res. 39 (2014), no. 3, 841-850. MR 3247006

[10] Ryogo Hirota, Nonlinear partial difference equations. V. Nonlinear equations reducible to linear equations, J. Phys. Soc. Japan 46 (1979), 312-319.

[11] Go-O Ishikawa and Yoshinori Machida, Singularities of improper affine spheres and surfaces of constant Gaussian curvature, Internat. J. Math. 17 (2006), no. 3, 269-293. MR 2215151

[12] O. V. Kaptsov and Yu. V. Shan' ko, Multiparametric solutions of the Tzitzeica equation, Differ. Uravn. 35 (1999), no. 12, 1660-1668, 1726. MR 1774990

[13] Shimpei Kobayashi, Nonlinear d'Alembert formula for discrete pseudospherical surfaces, J. Geom. Phys. 119 (2017), 208-223. MR 3661533

[14] Igor Moiseevich Krichever, An analogue of the d'Alembert formula for the equations of a principal chiral field and the sine-Gordon equation, Dokl. Akad. Nauk SSSR 253 (1980), no. 2, 288-292. MR 581396 
[15] A. Martínez, Improper affine maps, Math. Z. 249 (2005), no. 4, 755-766. MR 2126213

[16] Nozomu Matsuura and Hajime Urakawa, Discrete improper affine spheres, J. Geom. Phys. 45 (2003), no. 1-2, 164-183. MR 1949349

[17] Alexander V. Mikhailov, The reduction problem and the inverse scattering method, Physica D: Nonlinear Phenomena 3 (1981), no. 1, 73 - 117.

[18] Daisuke Nakajo, A representation formula for indefinite improper affine spheres, Results Math. 55 (2009), no. 1-2, 139-159. MR 2546487

[19] J. J. C. Nimmo and R. Willox, Darboux transformations for the two-dimensional Toda system, Proc. Roy. Soc. London Ser. A 453 (1997), no. 1967, 2497-2525. MR 1490247

[20] Franz Pedit and Hongyou Wu, Discretizing constant curvature surfaces via loop group factorizations: the discrete sine- and sinh-Gordon equations, J. Geom. Phys. 17 (1995), no. 3, 245-260. MR 97a:39022

[21] Helmut Pottmann, Sigrid Brell-Cokcan, and Johannes Wallner, Discrete surfaces for architectural design, Curve and surface design: Avignon 2006, Mod. Methods Math., Nashboro Press, Brentwood, TN, 2007, pp. 213-234. MR 2335142

[22] Andrew Pressley and Graeme Segal, Loop groups, Oxford Mathematical Monographs, The Clarendon Press, Oxford University Press, New York, 1986, Oxford Science Publications. MR 900587

[23] W. K. Schief, Hyperbolic surfaces in centro-affine geometry. Integrability and discretization, Chaos Solitons Fractals 11 (2000), no. 1-3, 97-106, Integrability and chaos in discrete systems (Brussels, 1997). MR 1729560

[24] C. Scimiterna, B. Grammaticos, and A. Ramani, On two integrable lattice equations and their interpretation, J. Phys. A 44 (2011), no. 3, 032002, 6. MR 2749061

[25] Georges Tzitzéica, Sur une nouvelle classe de surfaces, C. R. Acad. Sci. 144 (1907), 1257-1259.

[26] _ Sur une nouvelle classe de surfaces, C. R. Acad. Sci. 146 (1908), 165-166.

[27] _ Sur une nouvelle classe de surfaces, Rend. Circ. Mat. Palermo 25 (1908), 180-187.

[28] _ Sur une nouvelle classe de surfaces, Rend. Circ. Mat. Palermo 28 (1909), 210-216.

[29] _ Sur une nouvelle classe de surfaces, C. R. Acad. Sci. 150 (1910), 955-956.

[30] Sur une nouvelle classe de surfaces, C. R. Acad. Sci. 150 (1910), 1227-1229.

[31] A. V. ' Ziber and A. B. ` Sabat, The Klein-Gordon equation with nontrivial group, Dokl. Akad. Nauk SSSR 247 (1979), no. 5, 1103-1107. MR 550472

Department of Mathematics, Hokkaido University, Sapporo 060-0810, Japan

E-mail address: shimpei@math.sci.hokudai.ac.jp

Department of Education and Creation Engineering, Kurume Institute of Technology, KuRUME 830-0052, JAPAN

E-mail address: nozomu@kurume-it.ac.jp 